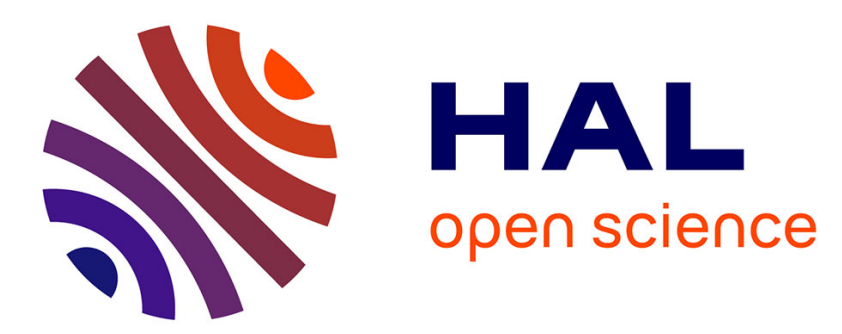

\title{
A mobile high-resolution gamma camera for therapeutic dose control during radionuclide therapy
}

Carlotta Trigila, Marc-Antoine Verdier, Laurent Pinot, Francoise Bouvet, Tiffany Mélanie Beaumont, David Broggio, Aurelie Desbree, Laurent Menard

\section{- To cite this version:}

Carlotta Trigila, Marc-Antoine Verdier, Laurent Pinot, Francoise Bouvet, Tiffany Mélanie Beaumont, et al.. A mobile high-resolution gamma camera for therapeutic dose control during radionuclide therapy. Phys.Med.Biol., 2022, 67 (3), pp.035011. 10.1088/1361-6560/ac4c31 . hal-03559624

\section{HAL Id: hal-03559624 \\ https://hal.science/hal-03559624}

Submitted on 23 Feb 2022

HAL is a multi-disciplinary open access archive for the deposit and dissemination of scientific research documents, whether they are published or not. The documents may come from teaching and research institutions in France or abroad, or from public or private research centers.
L'archive ouverte pluridisciplinaire HAL, est destinée au dépôt et à la diffusion de documents scientifiques de niveau recherche, publiés ou non, émanant des établissements d'enseignement et de recherche français ou étrangers, des laboratoires publics ou privés. 
PAPER • OPEN ACCESS

\section{A mobile high-resolution gamma camera for therapeutic dose control during radionuclide therapy}

To cite this article: Carlotta Trigila et al 2022 Phys. Med. Biol. 67035011

View the article online for updates and enhancements.

\section{You may also like}

- Biokinetics and dose assessment after
$\frac{\text { iodine intake in a thyroidectomised rat }}{\text { model }}$
Meriem Mezaguer-Lekouaghet, Maamar
Souidi, Abdewahab Badreddine et al.
- Laser-induced fluorescence method for
$\frac{\text { on-line molecular isotopoloques of iodine- }}{127 \text {, iodine-129, iodine-131 detected in }}$
$\frac{\text { gaseous media using a tunable diode }}{\text { laser }}$
S V Kireev, S L Shnyrev and I V
Sobolevsky
- The limit of detection in scintigraphic
$\frac{\text { imaging with I-131 in patients with }}{\text { differentiated thyroid carcinoma }}$
H Hänscheid, M Lassmann, A K Buck et
al.

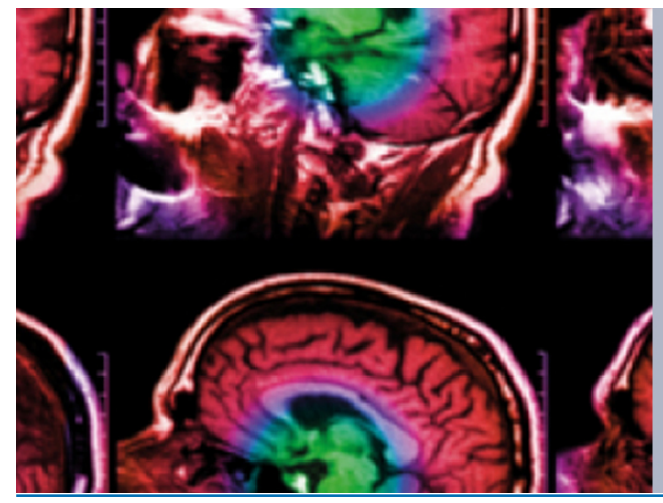

\section{IPEM IOP}

Series in Physics and Engineering in Medicine and Biology

Your publishing choice in medical physics,

biomedical engineering and related subjects.

Start exploring the collection-download the first chapter of every title for free.

This content was downloaded from IP address 81.194.12.18 on 23/02/2022 at 15:03 


\section{A mobile high-resolution gamma camera for therapeutic dose control during radionuclide therapy}

RECEIVED

9 November 2021

REVISED

29 December 202

ACCEPTED FOR PUBLICATION

17 January 2022

PUBLISHED

2 February 2022

Original content from thi work may be used under the terms of the Creative

Commons Attribution 4.0 licence.

Any further distribution of this work must maintain

attribution to the author(s) and the title of the work, journal citation and DOI.

\author{
Carlotta Trigila ${ }^{1}\left([)\right.$, Marc-Antoine Verdier ${ }^{1,2} \oplus$, Laurent Pinot $^{1}$, Françoise Bouvet $^{1}$, Tiffany Beaumont ${ }^{1}$, \\ David Broggio ${ }^{3}$, Aurelie Desbrée ${ }^{3}$ and Laurent Ménard ${ }^{1,2}$ (c) \\ 1 Université Paris-Saclay, CNRS/IN2P3, IJCLab, F-91405 Orsay, France \\ Université de Paris, IJCLab, 91405 F-Orsay, France \\ 3 Laboratory of Internal Dose Assessment, IRSN, Fontenay-aux-roses, France \\ E-mail: menard@imnc.in2p3.fr
}

Keywords: high resolution gamma-camera, radionuclide therapy, dosimetry, therapeutic dose control

\section{Abstract}

Objective. Molecular radiotherapy is the most used treatment modality against malign and benign diseases of thyroid. In that context, the large heterogeneity of therapeutic doses in patients and the range of effects observed show that individualized dosimetry is essential for optimizing treatments according to the targeted clinical outcome. Approach. We developed a high-resolution mobile gamma camera specifically designed to improve the quantitative assessment of the distribution and biokinetics of ${ }^{131} \mathrm{I}$ at patients's bedside after treatment of thyroid diseases. The first prototype has a field of view of $5 \times 5 \mathrm{~cm}^{2}$ and consists of a high-energy parallel-hole collimator made of 3D-printed tungsten, coupled to a $6 \mathrm{~mm}$ thick $\mathrm{CeBr}_{3}$ scintillator readout by an array of silicon photomultiplier detectors. The intrinsic and overall imaging performance of the camera was evaluated with ${ }^{133} \mathrm{Ba}$ and ${ }^{131} \mathrm{I}$ sources. In order to test its quantification capability in realistic clinical conditions, two different 3Dprinted thyroid phantoms homogeneously filled with ${ }^{131}$ I were used. Both single view and conjugate view approaches have been applied, with and without scatter correction technique. Main Results. The camera exhibits high imaging performance with an overall energy resolution of $7.68 \pm 0.01 \%$, a submillimetric intrinsic spatial resolution of $0.74 \pm 0.28 \mathrm{~mm}$ and a very low spatial distortion $0.15 \pm 0.10 \mathrm{~mm}$. The complete calibration of the camera shows an overall spatial resolution of $3.14 \pm 0.03 \mathrm{~mm}$ at a distance of $5 \mathrm{~cm}$ and a corresponding sensitivity of $1.23 \pm 0.01 \mathrm{cps} / \mathrm{MBq}$, which decreases with distance and slightly changes with source size due to the influence of scattering. Activity recovery factors better than $97 \%$ were found with the thyroid phantoms. Significance. These preliminary results are very encouraging for the use of our camera as a tool for accurate quantification of absorbed doses and currently motivates the development of a fully operational clinical camera with a $10 \times 10 \mathrm{~cm}^{2}$ field of view and improved imaging capabilities.

\section{Introduction}

Molecular radiotherapy (MRT) is a localized radiation treatment modality based on the internal delivery of a radiolabelled vectors, known as radiopharmaceutical, for the treatment of benign, malign and inflammatory conditions. MRT is currently rapidly evolving thanks to the joint development of new tumor-seeking biomarkers and innovative radionuclides, which allow to selectively deliver cytotoxic radiation to cells while causing minimal toxicity to surrounding healthy tissues. Among more than 35000 patients treated each year in Europe, ${ }^{131} \mathrm{I}$ remains the flagship radionuclide for the treatments of benign and malign thyroid diseases ( $84 \%$ of patients treated) (Sjögreen Gleisner et al 2017). Other applications are now available in clinic, as the therapy of neuroendocrine tumors using ${ }^{177} \mathrm{Lu} /{ }^{90} \mathrm{Y}$-somatostatin analogs $\left({ }^{177} \mathrm{Lu}\right.$-Dotatate or ${ }^{90} \mathrm{Y}$-Dotatoc), the treatment of prostate cancer metastasized to bones using ${ }^{223} \mathrm{Ra}$-dichloride (Xofigo ${ }^{\circledR}$ ) and the intra-arterial treatments of unresectable primary hepatocarcinoma and metastases with ${ }^{90} \mathrm{Y}$-microspheres (SIR-Spheres ${ }^{\oplus}$ or TheraSpheres ${ }^{\oplus}$ ) (Ting et al 2009, Yeong et al 2014). New radiopharmaceuticals, including agents that target tumor-associated 
antigens over-expressed in different malignant tissues are currently tested in clinical trials, such as PSMA for the treatment of prostate cancer (Eyben et al 2018). Because of the higher relative biological effectiveness of alpha radiation, the interest in the development of therapies involving alpha emitters, such as ${ }^{211} \mathrm{At},{ }^{225} \mathrm{Ac},{ }^{227} \mathrm{Th},{ }^{213} \mathrm{Bi}$, and ${ }^{212} \mathrm{~Pb}$, is also rapidly growing and first clinical trials show very promising results (Marcu et al 2018).

One of the major challenges in the use of MRT is the individual determination of the absorbed dose in target regions and organs-at-risk for both treatment planning and therapeutic dose verification. The need of individualized dosimetry relies both on the proof that different absorbed doses can be delivered from fixed activities, given that each patient has different radiopharmaceutical uptakes and clearance half-times (Smith and Wilson 1967, Eary et al 1997) and on the increasing evidence of the correlation between the dose delivered to patients and the treatment response, both in terms of therapeutic effects and toxicity (Stabin and Brill 2008, Salvatori and Luster 2010, Strigari et al 2014, Garin et al 2017). The objective of individualized dosimetry, as mandated by the European Council Directive 2013/59 (European Council Directive 2013), is to maximize the probability of a successful treatment response while maintaining the risk of toxicity to organs-at-risk at justifiable levels, as is commonly done in external radiotherapy or brachytherapy. In MRT, the determination of absorbed dose is based on the formalism of the medical internal radiation dose (MIRD), which relies on the quantitative measurement of the radiopharmaceuticals biodistribution over time (Siegel et al 1999). The maximal activity that could be administered to each specific patient, according to the treatment objectives and the tolerance doses to organs-at-risk, is first estimated from the calculation of the absorbed dose during a pre-treatment dose assessment. The post-treatment verification of the absorbed dose delivered to targeted and non-targeted tissues is also essential to refine the treatment planning. Indeed, it allows correlations to be established with the dose predicted by the pre-treatment study as well as with the observed clinical outcomes, both in terms of disease control and toxicity to organs-at-risk.

Despite its obvious value, personalized dosimetry-based treatment is still not yet well established in MRT and remains controversial: treatment planning based on the calculation of absorbed doses is rarely performed and post-treatment dosimetry verification is the exception rather than the rule (Strigari et al 2014). The majority of treatments performed in Europe are prescribed using different fixed-activity protocols, mainly tailored to patient anatomy (weight and body surface) or adjusted to the diagnosis or stage of the disease (Sjögreen Gleisner et al 2017). The lack of standardization across centers, and consequently the absence of large clinical trials that could prove the effectiveness of dosimetry in predicting therapy outcomes, is a major limitation in its use (O’Connor et al 1979, Flux et al 2006, Kesner and Bodei 2018, Lassmann and Eberlein 2018). Moreover, the implementation of personalized dosimetry is also hindered by a number of challenges that should be addressed. The first one is the accurate quantification of radionuclide biokinetics, considered as the largest contributor to absorbed dose uncertainties (Dewaraja et al 2012). At present, its determination is mainly performed using nonimaging measurements (counting tissue biopsies, blood or feces samples and external counting probes). Much more accurate results can be obtained using quantitative imaging performed with planar cameras or SPECT tomographic systems, by converting the counts recorded in images into an absolute value of activity. However, the use of imaging devices is currently limited by the fact that the performance of most clinical cameras is not optimized for imaging with high energy and high activity gamma-emitters, both in terms of spatial and energy responses, sensitivity and counting capability. In particular, their low spatial resolution (typical values ranging between 10 and $15 \mathrm{~mm}$ at $10 \mathrm{~cm}$ for $364 \mathrm{keV}$ gamma rays) reinforces the partial-volume effect and introduces large errors in quantifying the absorbed dose distribution for small targets (sizes less than 2-3 times the spatial resolution of the system) or organs where the radiopharmaceutical uptake is heterogeneous (Stabin and Brill 2008, van Gils et al 2016, Lassmann and Eberlein 2018). Dosimetry also poses logistical and practical challenges, as many images must be acquired at different times after treatment delivery. This automatically involves more steps than in a single diagnostic nuclear medicine examination (Flux et al 2018). Nowadays, the collection of images must therefore be limited to a few points in time, which leads to a lack of accuracy in the definition of the time-activity curve and therefore adds large uncertainties on the calculation of the absorbed dose (Dewaraja et al 2012, Eberlein et al 2017).

In that context, the objective of this work is to develop a high-resolution mobile gamma camera specifically designed to improve the individual quantitative assessment of the distribution and biokinetics of radiopharmaceuticals in target regions and organs-at-risk after treatment administration. The compactness and mobility of the camera will offer the possibility to improve image resolution by reducing the distance between the detector and the targeted structures and optimizing their detectability by adjusting the angular view. Its availability and the possibility of using it at the patient's bedside (e.g. in the isolation room) will also be compatible with an accurate temporal sampling of the tracers's biokinetics, a key parameter for the quantification of the absorbed doses. This need is reinforced by the fact that in many nuclear medicine departments, the number of multi-purpose wide-field cameras is expected to decrease in favor of PET systems. Unlike conventional large field-of-view gamma cameras, the device will also be optimized to meet the specific needs of imaging with high-energy gamma rays (above $300 \mathrm{keV}$ ) and high rate of photon fluence. The final objective is to achieve a system spatial resolution ranging between 3 and $5 \mathrm{~mm}$ FWHM at a $5 \mathrm{~cm}$ source distance, 

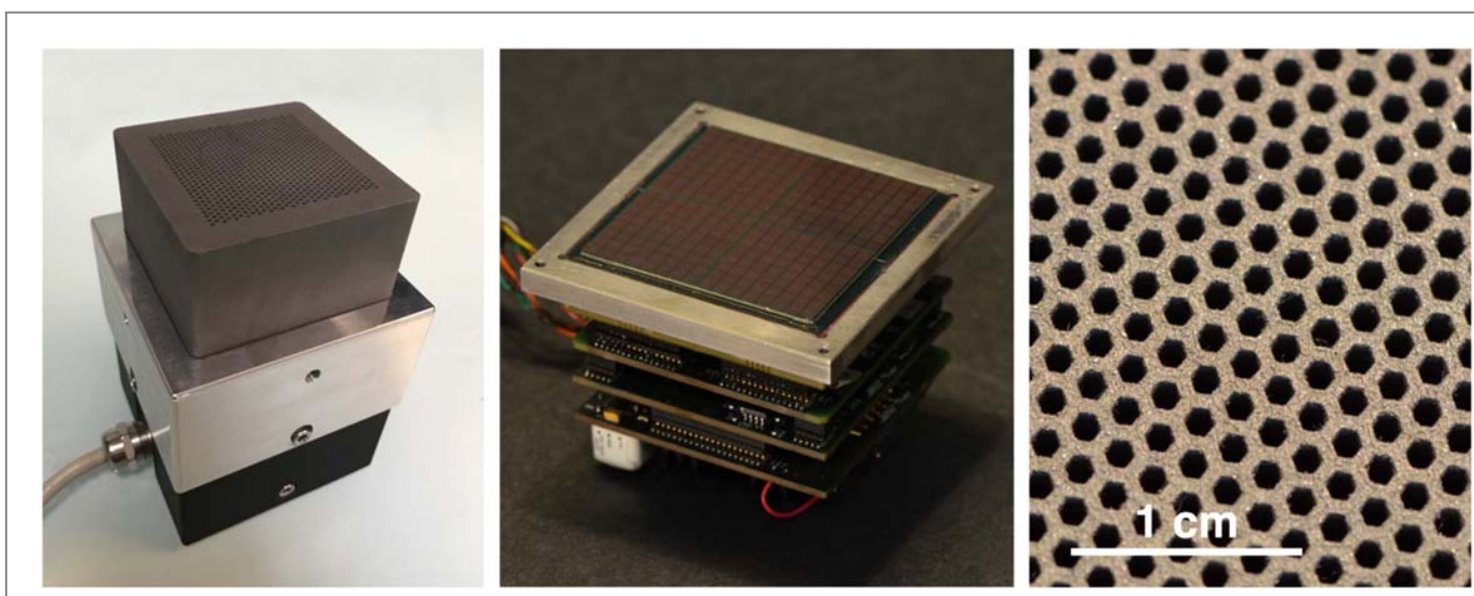

Figure 1. The first prototype of the mobile gamma camera (left), the photodetector and miniaturized readout electronics (middle) and the hole structure of the high-energy parallel-hole collimator made of 3D-printed tungsten (right).

much better than that of conventional gamma cameras equipped with high-energy collimator and suited to the maximum path in tissue of electrons emitted by ${ }^{131} \mathrm{I}$, an energy resolution less than $8 \%$ in order to minimize the contamination from high-energy scattered events and a maximum counting capability above $200 \mathrm{kcps}$. The high counting capability of the camera will greatly reduce the effect of dead time on activity estimates and will open the door to early imaging, even shortly after several GBq of activity has been administered (Dewaraja et al 2013).

We chose to focus our first application on the treatment of benign and malign thyroid diseases with ${ }^{131} \mathrm{I}-\mathrm{NaI}$, which has been used since the 1940s to ablate thyroid remnant or inoperable advanced differentiated cancer and to treat patients with hyperthyroidism (Sjögreen Gleisner et al 2017). Due to its selective tropism for thyroid tissue, the use of ${ }^{131}$ I provides high treatment efficiency with low toxicity to healthy tissue, although these thyroid's therapies are mainly prescribed with fixed activities (Stabin and Brill 2008, Vija Racaru et al 2017). However, the importance of the dosimetric approach to the treatment of thyroid disease is still emphasized to optimize the administered activity according to the desired clinical outcome (destruction of normal and tumor residues and lymph node metastases for differentiated thyroid cancers and euthyroidism or hypothyroidism for benign thyroid diseases) or to limit undesired side effects of irradiation on high-risk organs (including salivary and lacrimal glands) and long-term consequences (Smith and Wilson 1967, Lassmann et al 2008, Stokkel et al 2010). One of the main challenges for individualized treatment planning and post-treatment verification of the absorbed doses is to develop a reasonably simple, cost-effective and acceptable method for measuring ${ }^{131} \mathrm{I}$ biokinetics in thyroid, tumor remnants, metastatic lymph nodes or salivary glands (Vija Racaru et al 2017). Dead-time and partial-volume effects are particularly important for imaging with ${ }^{131}$ I due to the large number of high-energy scattered events (from 637 and $723 \mathrm{keV}$ photons) that contribute to the dead time and low contrast of images obtained with conventional gamma cameras (Dewaraja et al 2013).

This article describes the design and intrinsic performance characterization of the first feasibility prototype of the mobile gamma camera with a field of view of $5 \times 5 \mathrm{~cm}^{2}$. The calibration of the overall system response (spatial resolution and sensitivity) as a function of the source-to-collimator distance is presented. This calibration has been performed by using capillary tubes and thin cylindrical sources with different diameters placed in air and in tissue-equivalent medium, all filled with ${ }^{131}$ I. Finally, a preliminary assessment of the ability of the camera to quantify ${ }^{131}$ I activity is reported according to MIRD pamphlet 16 (Dewaraja et al 2012) with 3Dprinted thyroid phantoms.

\section{Materials and methods}

\subsection{Design of the camera}

A photograph of the first $5 \times 5 \mathrm{~cm}^{2}$ field of view (FOV) camera prototype is shown in figure 1 (left). In order to achieve the desired objectives in terms of performance and ergonomics, the mobile gamma-camera consists of a high-energy parallel-hole tungsten collimator, made by 3D printing (M\&I Materials Ltd), coupled to a monolithic $\mathrm{CeBr}_{3}$ inorganic scintillator, readout by an array of Silicon Photomultiplier (SiPMs) detectors. The overall dimensions of the camera are $12.6 \times 8.6 \times 8.6 \mathrm{~cm}^{3}$ and it weights is $4.54 \mathrm{~kg}$ (including the $3.76 \mathrm{~kg}$ of the collimator). 


\subsubsection{Photodetection system and readout electronics}

The SiPMs array consists of four Hamamatsu S13361-3050NE-08 monolithic arrays covering an overall area of $51.6 \times 51.6 \mathrm{~mm}^{2}$ and a sensitive area of $48 \times 48 \mathrm{~mm}^{2}$ (figure 1 (middle)). Each array is composed of $8 \times 8$ pixels with a $3 \times 3 \mathrm{~mm}^{2}$ effective photosensitive area, a cell size of $50 \mu \mathrm{m}$ and a dead space of $0.2 \mathrm{~mm}$ between pixels. The optical window of the arrays is made of an epoxy resin with a refractive index of 1.55 . The four arrays are mounted on a single PCB containing passive elements (resistance, capacitances) for bias and four digital thermal sensors, on the backside. The miniaturized readout electronics (figure 1 (middle)) contains eight 32-channels EASIROC ASICs with fully analogical front end electronics dedicated to gain trimming and SiPMs readout (Dinu et al 2015). The analog charge is digitized using an external two-channel ADCs with 12-bits dynamic performance and a sampling rate of $2 \mathrm{MHz}$. The front-end module is mounted on three 256-channels motherboards combining an FPGA (Altera Cyclone III), a FTDI FT2232H (USB protocol 2.0 Hi-Speed, 480 $\mathrm{Mbit} / \mathrm{s}$ ) for the communication with a PC and the power supplies (including the DC/DC convertor, which delivers the high voltage to the SiPMs). The digitization of the analog signals takes around $12 \mu$ s for the 256 channels. The data transfer through the USB lasts $8 \mu$ s and generates most of the dead time. The count loss at a frequency of $1 \mathrm{kHz}$ is $2 \%$ and follows a non-parallelizable behavior as a function of activity. The response uniformity of the SiPM arrays is improved by an 8-bit DAC $(0-2.5 \mathrm{~V})$ embedded at the ASIC input, which allows each SiPM polarization voltage to be individually adjusted. The four thermal sensors are connected to the FPGA, which adjusts in real-time the DC/DC converter as a function of the mean temperature in order to maintain a constant overvoltage on the SiPMs (accuracy of $35 \mathrm{mV}$ ) and, thus, to accurately offset the influence of temperature variation on SiPM performance, including intrinsic gain and photodetection efficiency. The photodetection system and readout electronics are held in an aluminum housing with external dimensions of $6.5 \times 6.5 \times 5 \mathrm{~cm}^{3}$.

\subsubsection{The scintillator}

$\mathrm{CeBr}_{3}$ has been chosen as the inorganic scintillator to be coupled to the SiPMs. Its high light output $(60 \gamma / \mathrm{keV})$, good energy resolution (4.6\% at $662 \mathrm{keV}$ with a PMTs) and fast response (18 ns) (Lecoq 2016) make it comparable to $\mathrm{LaBr}_{3}(\mathrm{Ce})$, but with a two times lower price. Two $51 \times 51 \mathrm{~mm}^{2} \mathrm{CeBr}_{3}$ samples with a thickness of $6 \mathrm{~mm}$ and $10 \mathrm{~mm}$ have been tested (Scionix). Both have a diffuse optical coating on the entrance surface and on the edges. Due to the hygroscopicity of the material, the tested samples were encapsulated by the producer in an aluminum housing with a $1.5 \mathrm{~mm}$ thick quartz light guide. They were optically coupled to the SiPMs array using an optical grease with a refractive index of 1.46 .

\subsubsection{The high-energy parallel-hole collimator}

The collimator has been designed to improve the quantitative assessment of small heterogeneous activity distributions during radioidodine treatment of malignant and benign thyroid diseases. Quantification with ${ }^{131} \mathrm{I}$ is much more difficult than with common nuclear medicine isotopes such as ${ }^{99 \mathrm{~m}} \mathrm{Tc}(140 \mathrm{keV})$ because of its high photopeak energy $(364 \mathrm{keV}, 81.5 \%)$ and its multiple high energy $\gamma$-ray emissions $(636.9 \mathrm{keV}$ and $722.9 \mathrm{keV}$, respectively $7.16 \%$ and $1.77 \%$ ), which could penetrate and scatter inside the collimator degrading both spatial resolution, image contrast and consequently, dose quantification.

The design of the collimator was optimized using GATE Monte Carlo simulations and an XCAT voxelized phantom (Jan et al 2004, Segars et al 2010). The main purpose was to achieve a high spatial resolution in order to minimize the partial volume effect, while reducing both scattered and penetration events. This has been done at the expense of sensitivity, but sensitivity is no longer a key parameter for the post-treatment verification of the absorbed dose due to the large amount of activity administered during the majority of radioiodine treatments (Luster et al 2008, Stokkel et al 2010). A $5.5 \mathrm{~cm}$ thick parallel-hole collimator made of tungsten with $1.1 \mathrm{~mm}$ hexagonal holes and $0.75 \mathrm{~mm}$ thick septa was finally chosen in order to achieve a $2 \mathrm{~mm}$ spatial resolution for a source placed $5 \mathrm{~cm}$ from the collimator (calculated from the standard deviation $\sigma$ of a Gaussian fit on the spatial profile of ${ }^{131}$ I line source) and an effective septal penetration of $7.5 \%$. The effective septal penetration is defined as the ratio between the scattered and penetration events outside a region of interest (ROI) with a radius of $2 \sigma$ drawn around the spatial peak profile of the line source and the total number of detected events. This criterion is much more restrictive than the usual 5\% maximum septal penetration accepted when optimizing collimator geometry and calculated in a simplistic way from the shortest path a gamma ray can travel through a single septum, which strongly underestimates the contribution of penetration for high-energy gamma rays (Mather 1957). The expected total efficiency of the collimator is $1.24 \times 10^{-5}$. The tungsten collimator was 3Dprinted with a selective melting method (M\&I Materials), which consists of using a high-power laser to fuse metallic powder grains in order to obtain the desired three-dimensional shape (figure 1(right)). 


\subsection{Intrinsic performances of the camera}

The intrinsic imaging performances of the camera have been evaluated in terms of energy resolution, intrinsic spatial linearity, spatial resolution and uniformity, using a $1 \mathrm{~mm}$ diameter ${ }^{133}$ Ba point source (Eckert \& Ziegler). All measurements were performed at $25^{\circ} \mathrm{C}$ inside a temperature controlled chamber. The temperature on the SiPMs was monitored by the acquisition software thanks to the four thermals sensors connected to the FPGA. The measurements were performed by applying an optimal overvoltage of $69 \mathrm{~V}$ on the SiPMs array. The preamplifiers gain, delay and trigger threshold of the electronics readout have been adjusted for each of the two configurations in order to optimize their energy resolution, to reduce the dynamic saturation and avoid any dead time due to the dark count rate. For each detected scintillation event, the 2D-position of the interacting gammaray in the crystal plate was reconstructed using nonlinear least squares based on the iterative LevenbergMarquardt algorithm minimizing the difference between the intensity values of the 256 pixels of the SiPMs array and the Scrimger-Backer model of the light distribution (Mathai and Katiyar 1996). The deposited energy by the incident gamma ray was assumed to be proportional to the sum of the light intensity in all pixels. When all data are displayed in real-time on the acquisition software with this reconstruction algorithm, the maximum counting rate of the camera is $5 \mathrm{kcps}$. Mean values and standard deviations have been measured in the useful field of view (UFOV), corresponding to the complete FOV of the camera, in the Central FOV (CFOV, 75\% of the FOV), defined according to NEMA standards (Association 2018), and in the complementary region of the $\mathrm{CFOV}$, the $\overline{\mathrm{CFOV}}$, in order to highlight the intrinsic performances close to crystal edges.

The energy resolution was evaluated with the ${ }^{133} \mathrm{Ba}$ source collimated with a $4 \mathrm{~mm}$ diameter single-hole tungsten collimator. The source was mounted on a 3-axis motorized platform with $2 \mu \mathrm{m}$ position accuracy and a scan of the whole FoV with $10 \times 10$ uniformly distributed positions has been performed, moving it with $5 \mathrm{~mm}$ steps. Only reconstructed events inside a $5 \mathrm{~mm}$ diameter circular ROI centered on the source image have been considered. The FWHM energy resolution and photopeak location were calculated at each position from a double Gaussian fit performed on the full-energy peaks of the energy spectra corresponding to the principal ${ }^{133} \mathrm{Ba}$ emission at $356 \mathrm{keV}(62 \%)$ and its secondary peak at $383 \mathrm{keV}(8.94 \%)$. The linearity of the energy response and the keV-per-channel calibration factor were estimated from the linear fit on the photopeak positions measured with the $80 \mathrm{keV}$ and $356 \mathrm{keV}$ gamma-rays emissions of the ${ }^{133} \mathrm{Ba}$ source and the $122 \mathrm{keV}$ gamma-rays emission of a ${ }^{57} \mathrm{Co}$ source. In addition to the energy resolution map, the relative light collection, defined as the relative displacement of the photopeak with respect to the highest position of the photopeak in the field of view, was also computed as a $10 \times 10$ position array. The inverse of this map was used to correct for spatial variations in the light collection to improve the uniformity of the energy response. This procedure was applied to the data obtained from a flood-field irradiation with the ${ }^{133} \mathrm{Ba}$ source placed at $25 \mathrm{~cm}$ from the scintillator. A corrected total spectrum was then obtained, from which the overall system energy resolution was calculated.

The flood-field corrected images were also used to estimate the system spatial uniformity. A $64 \times 64$ pixels image, with an average number of detected events per pixel greater than 8000 , was produced and smoothed with a $3 \times 3$ kernel Gaussian filter. Both integral and differential uniformity have been calculated. The integral uniformity (IU) has been obtained as the ratio of the standard deviation of the counts per pixel to the mean counts by removing the edge pixels. The differential uniformity (DU) was calculated from ten IU values for each pixel, corresponding to the IU in the 2, 4, 6, 8 and 10 nearest neighbor pixels in each pixel's row and column. These values were collected and used to produce a histogram for differential uniformities. The mean and dispersion of this distribution were used as a measure of DU (Bhatia et al 2015).

The intrinsic spatial performances were estimated through a $3 \mathrm{~mm}$ step-scanning of the whole FOV $(17 \times 17$ spots) performed with the ${ }^{133}$ Ba source collimated by a $0.5 \mathrm{~mm}$ diameter single-hole tungsten collimator fixed on the motorized platform. In order to have more spatial information near the edges of the crystal, additional scan lines were performed between the two outermost lines of scan spots all around the field of view. All properties were estimated with an energy selection of $15 \%$ around the $356 \mathrm{keV}$ photopeak of the ${ }^{133} \mathrm{Ba}$ spectra (329-383 keV). A two-dimension Gaussian fit was applied to the two-dimension histogram of the reconstructed positions in order to accurately evaluate the $x_{i, r e c}$ and $y_{j, \text { rec }}$ coordinates of the center of the distribution for each scan position $(i, j)$, as well as the two associated standard deviations of the point spread function $\sigma_{x_{i}}$ and $\sigma_{y_{j}}$. The horizontal local spatial linearity $L_{x_{i}}$, corresponding to the spot $i$ with an horizontal mechanical position $x_{i, m e c}$, was calculated using its reconstructed position $x_{i, r e c}$ and the mechanical and reconstructed positions of its adjacent spots, $x_{i \pm 1, m e c}$ and $x_{i \pm 1, \text { rec }}$ respectively, according to the following formula:

$$
L_{x_{i}}=\frac{1}{2}\left(\frac{x_{i, r e c}-x_{i-1, r e c}}{x_{i, m e c}-x_{i-1, m e c}}-\frac{x_{i+1, r e c}-x_{i, r e c}}{x_{i+1, m e c}-x_{i, m e c}}\right) .
$$

A similar formula was applied to obtain the vertical local linearity $L_{y_{i}}$. Any variation of $L$ from the unity reflects a local distortion that affects the spatial resolution. This spatial resolution $\mathrm{SR}_{i, x}$ along the $x$ direction for the iscan position $\left(x_{i, m e c}, y_{i, m e c}\right)$ was defined as the ratio between the FWHM of the point spread function along 
the $x$ direction obtained from $\sigma_{x_{i}}$ and the local linearity $L_{x_{i}}$. The spatial resolution along the $y$ direction, $\mathrm{SR}_{j, y}$, was obtained in the same way. Finally, the total intrinsic spatial resolution (SR) was calculated as the average value of $\mathrm{SR}_{i, x}$ and $\mathrm{SR}_{j, y}$ deconvolved by the collimator hole diameter. The results are represented in the forme of a map, where each box corresponds to the average of the local intrinsic spatial distortion measured for the scan positions associated with that region $\left(3 \times 3 \mathrm{~mm}^{2}\right)$. For each line ( $x$ direction) and column ( $y$ direction $)$, a linear fit was performed on the curves representing the reconstructed position as a function of the true mechanical position obtained for the 13 internal spots, in order to estimate a best-fit curve. These best-fits allowed to obtain the horizontal and vertical local intrinsic spatial distorsion (LISD and LISD $_{y}$ ), calculated as the horizontal (vertical) distance, in $\mathrm{mm}$, between the spot's reconstructed position $x_{i, r e c}\left(y_{i, r e c}\right)$ and the best-fit curve. A map of the local intrinsic spatial distortion was also performed, in which the direction of the local distortion for each scan position $(i, j)$ is given by a vector $\left(\operatorname{LISD}_{x}, \mathrm{LISD}_{y}\right.$ ) and its intensity by the norm of this vector. The maximum value of LISD $x$ and LISD $y$ are identified as the absolute linearity AL according to NEMA standards (Association 2018). These values were reported in both the UFOV and CFOV.

\subsection{Camera calibration for quantification}

A mandatory prerequisite for quantitative imaging dedicated to dosimetry-guided radionuclide therapy is the calibration of the imaging device. The calibration factor (system sensitivity that converts count rate to absolute activity in each pixel of the image) was shown to be the most important contribution to the overall uncertainty in the dosimetric procedure, besides partial volume correction (Siantar et al 2002). The calibration of the camera was performed using ${ }^{131}$ I sources in liquid solution. The system response was measured, in terms of spatial resolution and sensitivity (count rate per unit activity), using a capillary tube and five thin cylindrical source phantoms with different diameters placed in air and in tissue-equivalent medium, as a function of the source-tocollimator distance. All measurements have been done at room temperature by using the temperaturecompensated circuit. The source activities were chosen so that the effect of dead time on the activity estimates was negligible. Considering the low geometrical efficiency of our high resolution collimator and the stopping power of the scintillator, the total number of detected events, including scattering background, is around $12 \mathrm{cps}$ per Mbq of source activity. According to the dead time of the readout electronics, the expected count loss is $1 \%$ for a $50 \mathrm{MBq}$ source and the maximum counting rate of the camera ( $5 \mathrm{kcps}$ ) corresponds to an activity of $400 \mathrm{MBq}$. Given these limitations, the activities of the source phantoms used for the calibration of the camera were set below $50 \mathrm{MBq}$.

\subsubsection{System spatial resolution}

The spatial resolution was measured using a glass capillary tube with an internal diameter of $0.86 \pm 0.05 \mathrm{~mm}$ (Clark Electromedical instruments GC150F-10) filled with a water solution of ${ }^{131}$ I. The tube was tilted with respect to the $X$ axis of the camera in order to provide an oversampling of the line profiles with different sourcecollimator holes relative positions. The line source was moved from 1 to $15 \mathrm{~cm}$ from the collimator in $2 \mathrm{~cm}$ steps.

Events were selected in an energy window of $15 \%$ centered on the ${ }^{131}$ I principal gamma emission peak $(337-391 \mathrm{keV})$ and reconstructed in $128 \times 128$ pixels planar images with the least-squares method (see section 2.2). Images were then processed with a filter specifically designed to remove the high frequency artifacts produced by the high-energy collimator (Garcia and Barquero 2017). The number of counts in a given ROI was shown to remain invariant before and after its application. Figure 2 shows a schematic summary of the filtering protocol applied to a $128 \times 128$ image. The sum of the profiles through the filtered image of the line source was measured along the $X$ and $Y$ directions after their translation to account for the angular offset of the source. The FWHM spatial resolution of the system was calculated from the profile of the line spread function with a doubleGaussian fit in order to account for both the main peak, consisting mainly of geometric events, and the tails, including penetrating and scattering events.

\subsubsection{System sensitivity}

The sensitivity was measured using five 3D-printed cylindrical source phantoms (VeroClear, $\mu=0.116 \pm 0.002$ $\mathrm{cm}^{-1}$ ) with a diameter of 4,3,2,1 and $0.5 \mathrm{~cm}$ and a thickness of $3 \mathrm{~mm}$. The cylindrical sources were filled with ${ }^{131} \mathrm{I}$ in liquid solution. The source activity was controlled with an activimeter (MEDI 405, accuracy of $\pm 3 \%$ ) and ranged from 8.9 to $55.5 \mathrm{MBq}$ for the smallest and largest source, respectively. For each cylinder, $20 \mathrm{~min}$ acquisitions were performed at 8 different distances from the collimator surface, from 1 to $15 \mathrm{~cm}$ with a $2 \mathrm{~cm}$ step. Measurements were carried out with and without an absorbing medium, in the form of $5 \times 5 \times 1 \mathrm{~cm}^{3}$ bricks of $\operatorname{ABS}\left(\rho=0.97 \mathrm{~g} \mathrm{~cm}^{-3}\right)$. The image reconstruction protocol was identical to the one used for the spatial resolution measurement (figure 2). The number of counts inside ROIs defined on the images of the cylindrical sources divided by the acquisition time and the source activity, corrected for its decay over time, was recorded as a measure of the system sensitivity (cps/MBq). 

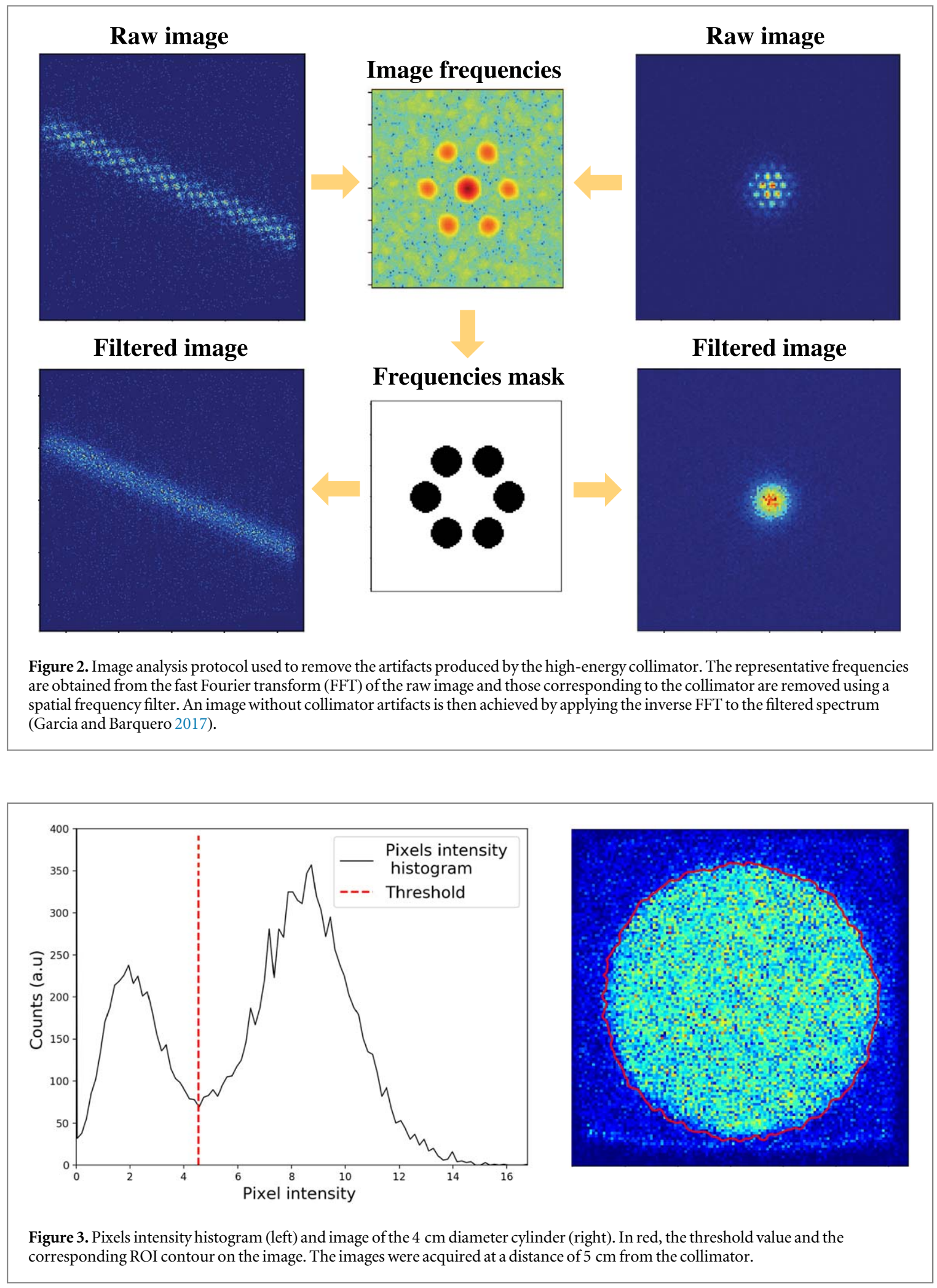

Quantitative measurements can be strongly dependent on the method used to define the regions of interest (ROI) around the lesions. The criteria required to define the ROI around the sources and to measure the detected counts should involve the selection of all or most of the geometric events and strongly depends on the size of the volume being studied, i.e. whether its dimensions are smaller or larger than the system spatial resolution. In order to define ROIs encompassing non-standard-shape lesions, threshold-methods are commonly used (Lin et al 2011). They consist in hiding the pixels of the image having an intensity value lower than a threshold value. In this work, the threshold values were defined from the pixels intensity histograms (figure 3 ). These histograms are characterized by two distinct intensity distributions: the first one, lower in pixels intensity (low-intensity peak), mainly related to scatter and penetration contributions outside the source image, and the uptake peak, 


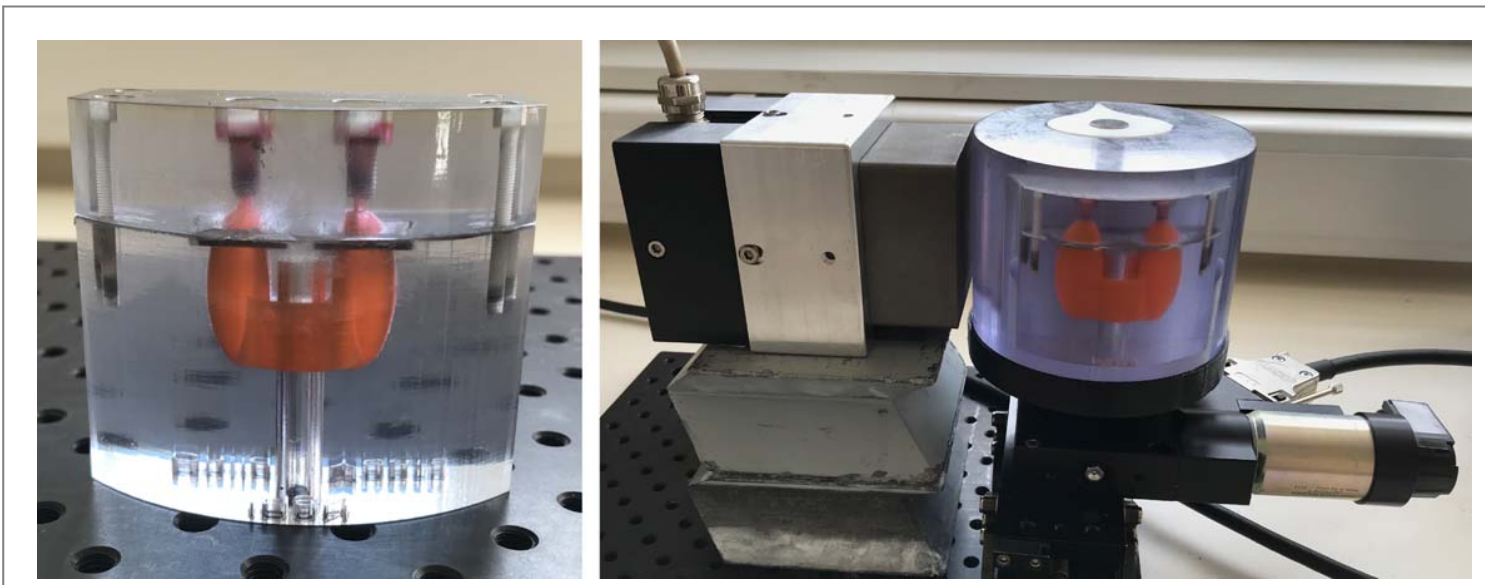

Figure 4. 5 years old (FANTHY5y) child 3D-printed thyroid phantom filled with ${ }^{131}$ I in liquid solution (left). Experimental set-up used for quantitative planar imaging with the thyroid phantoms (right). Sides views of the camera with the 15 years old child thyroid phantom (FANTHY15y) inserted in the neck phantom. The lead shielding around the camera is not shown on the picture.

higher in pixel intensity, mainly related to geometric events in the source image. Whatever the size of the source, the threshold was set at the minimum between the low-intensity peak and the uptake peak.

As already mentioned, ${ }^{131}$ I has two high-energy photon emissions at 637 and $723 \mathrm{keV}$, which can scatter and contaminate the $364 \mathrm{keV}$ photopeak window, resulting in lower image contrast, overestimation of system sensitivity and, therefore, in quantification bias. In planar imaging, this effect is commonly corrected through the implementation of the triple energy windows (TEW) scatter correction technique (Siegel et al 1999). This consists in estimating the fraction of scattered photons in the photopeak window to be subtracted from the total number of counts within it, using two additional windows, one below and one above the photopeak (Dewaraja et al 1998, Siegel et al 1999). Despite its practicality for clinical implementation, various studies have shown that the TEW scatter correction method could lead to a significant overestimation of the scatter contribution within the photopeak (van Gils et al 2016, Robinson et al 2016). Thus, the system sensitivity was estimated both without and with scatter correction, using two $7.5 \%$ wide energy windows (309-336.7 keV and 391.3-418.6 keV, respectively).

\subsection{Quantitative planar imaging for dosimetry during thyroid treatments}

\subsubsection{The $3 D$ thyroid phantoms}

The quantification ability of the camera was evaluated using two 3D-printed thyroid phantoms (thyroid lobes plus isthmus) (Beaumont et al 2017). Due to the limited field of view of the camera, phantoms representing 5 years old (FANTHY5y, figure 4 (left)) and 15 years old children (FANTHY15y, figure 4 (right)) were used. Their overall volumes are $3.2 \mathrm{ml}$ and $11.5 \mathrm{ml}$, for both lobes and isthmus, corresponding to a width of $2.2 \mathrm{~cm}$ and $4.5 \mathrm{~cm}$ and a height of 2.5 and $3.8 \mathrm{~cm}$, respectively. They are made of a tissue-equivalent polymeric resin (VeroClear, $\mu=0.116 \pm 0.002 \mathrm{~cm}^{-1}$ ). Both phantoms were inserted inside a 3D-printed neck phantom (VeroClear, $\mu=0.116 \pm 0.002 \mathrm{~cm}^{-1}$ for the neck adipose tissue and the spinal cord and VeroWhite for the vertebral column, $\mu=0.124 \pm 0.001 \mathrm{~cm}^{-1}$ ) (figure 4 (right)). The phantoms were designed following ICRP recommendations for human body volumes and the materials were chosen to accurately simulate the attenuation of biological tissues (Beaumont et al 2017). The neck has an overall diameter of $10.8 \mathrm{~cm}$ and a height of $8.4 \mathrm{~cm}$.

\subsubsection{Experimental protocol}

The thyroid phantoms were filled with ${ }^{131}$ I in a colored liquid solution. The activities, controlled with an activimeter (MEDI 405), were 52.06 $\pm 1.56 \mathrm{MBq}$ and 49.90 $\pm 1.50 \mathrm{MBq}$ for the FANTHY5y and FANTHY15y, respectively. As with the calibration study, these activities were chosen so that the effect of dead time on the activity estimates was negligible. The activity was also set as close as possible to realistic activities encountered in the treatment of hyperthyroidism. The complete phantom was positioned on a support that allowed it to be moved vertically and horizontally $( \pm 0.5 \mathrm{~mm})$ in order to ensure the centering of the thyroid lobes with the camera FOV and to rotate it using a motorized rotation stage $\left( \pm 0.02^{\circ}\right)$. The camera was inserted inside a $2 \mathrm{~cm}$ thick lead shielding in order to reduce scattered radiation that could bypass the collimator and enter the scintillator from the sides and back of the camera (total attenuation of $99.8 \%$ at $356 \mathrm{keV}$ ). The complete experimental set-up is shown in figure 4 (right). A distance of $3 \mathrm{~mm}$ was set between the neck phantom and the collimator. For each phantom, several planar images with different angular views were performed: $0^{\circ}$ (anterior), 

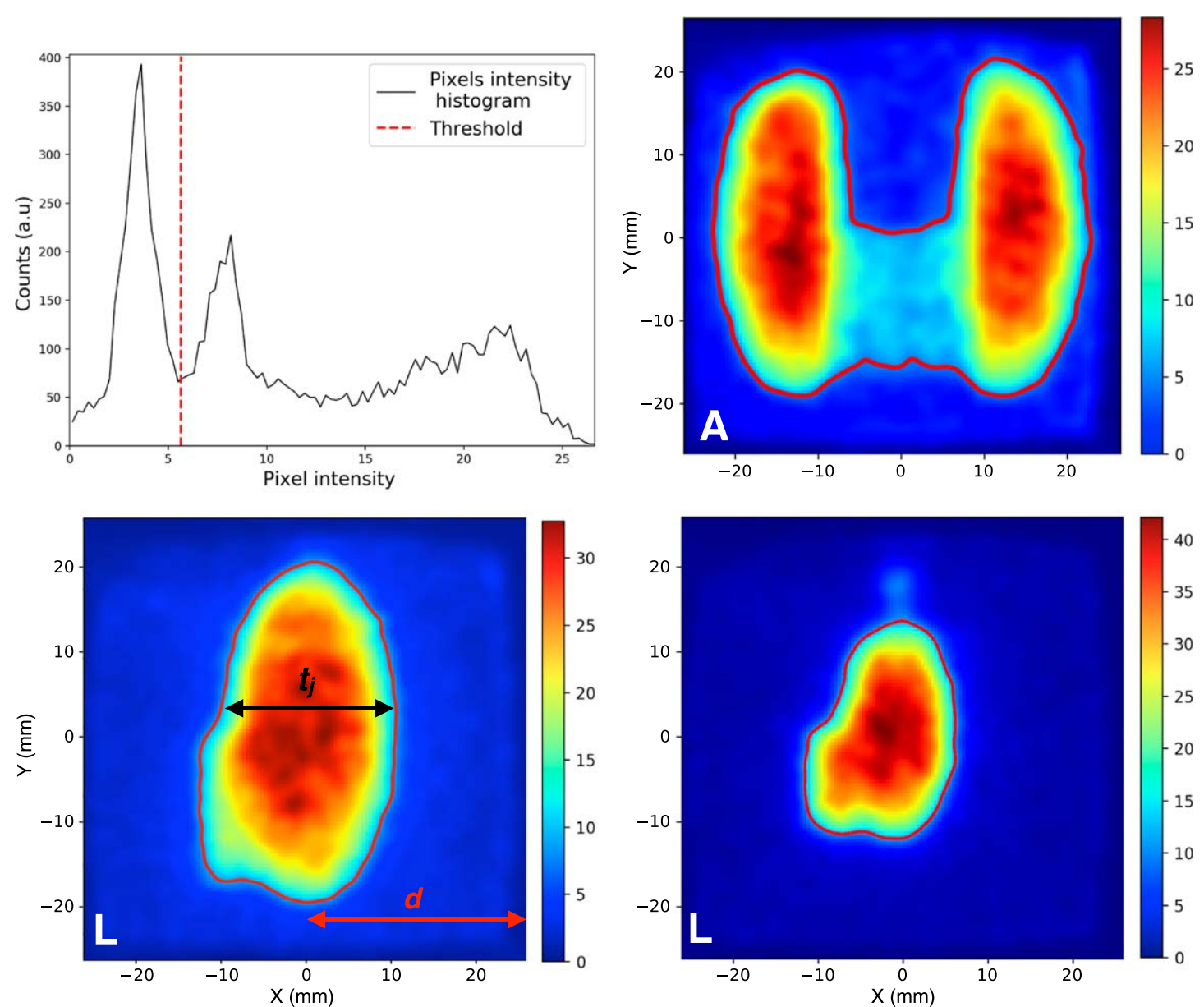

Figure 5. Pixels intensity histogram for the 15 years old child thyroid phantom (top left) together with the anterior image (A) and ROI contour defined using the threshold method (top right). The first peak of the histogram corresponds to the background noise outside the thyroid lobs, the second to the activity in the isthmus and the third to the lobes. Lateral images $(L)$ of the 5 (bottom left) and 15 (bottom right) years old children thyroid phantoms used to measure lobes thicknesses $\left(t_{j}\right)$ and to extrapolate their depth in the neck $(d)$.

$180^{\circ}$ (posterior) and $90^{\circ}$ (lateral). For each angular view, a 25 min acquisition time was set. All measurements were made at room temperature by using the temperature-compensated circuit. The image reconstruction and analysis protocols was identical to the one used for the calibration with the cylindrical phantoms, including the TEW scatter correction method. The definition of the ROI that should encompass the uptake in the thyroid images was performed using the threshold method. Figure 5 shows an example of the pixels intensity histogram and the corresponding ROI contour defined from the threshold value on the anterior image of the thyroid phantoms. All images are smoothed with a two-sigma Gaussian filter for better visualization.

The lateral images were used to estimate the depth and thickness of the thyroid in the neck, both useful for activity quantification. The camera has been positioned so that the edge of its field of view coincides with the edge of the neck. The depth and thickness of the lobes were estimated by applying the threshold method and extracting the information from the ROI around the lobes, as illustrated in figure 5 . The lobe thickness $t_{j}$ was defined as the mean width of the ROI calculated from five adjacent horizontal profiles. As visible from the images, the isthmus is clearly visible and has a significant impact on the central width of the ROI. To get rid of it, the five horizontal profiles were drawn just above the outgrowth. Finally, the depth $d$ of the lobes was defined as the horizontal distance between the right edge of the image and the centroid of the ROI. The measured values have been compared to those corresponding to the actual geometric characteristics of the phantoms.

\subsubsection{Planar imaging quantification methods}

The activity quantification was performed using both single-view (SV) planar images and the conjugate view (CV) approach, according to MIRD pamphlet 16 (Siegel et al 1999). In the single view method, the source activity $A_{j}$ in the thyroid was individually measured from the anterior or the posterior planar images (Siegel et al 1999). In a first approach, the counting rate within the ROI around the thyroid images, i.e. $I_{A}$ or $I_{P}$ for the $A$ and $P$ images respectively, was divided by the system calibration factor $C(\mathrm{cps} / \mathrm{MBq})$ measured without absorbing medium and by an attenuation factor in the form of $e^{-\mu d} . d$ is the thyroid-to-collimator distance when 
positioned at $0^{\circ}$ or $180^{\circ}$, estimated from the lateral planar images and from the known phantom-to-collimator distance. $\mu$ represents the theoretical attenuation coefficient of adipose tissue at $364 \mathrm{keV}\left(0.106 \mathrm{~cm}^{-1}\right.$ (Hubbell and Seltzer 2004)). In an alternative approach, $I_{A}$ or $I_{P}$ were only divided by the system calibration factor $C_{a b s}$ (cps/MBq) measured with an absorbing medium. The calibration factors $C_{a b s}$ and $C$ were extrapolated from the distance-sensitivity curve obtained with the cylindrical sources with and without absorbing medium. They correspond to the sensitivity values measured at a distance $d$ with the cylindrical source whose surface area was the closest to that of the ROI around the thyroid image.

The conjugate view method is the most commonly used protocol for in vivo radioactivity quantification with planar images. It uses two $180^{\circ}$ opposed images together with an orthogonal view to estimate the source organ thickness and position. When considering a source regions $j$ with negligible surrounding background activity, the activity $A_{j}$ can be calculated as:

$$
A_{j}=\sqrt{\frac{I_{A} I_{P}}{e^{\mu_{e} t_{n}}}} \frac{f_{j}}{C}
$$

where $I_{A}$ and $I_{P}$ are the conjugate-view counting rate and $e^{\mu_{e} t_{n}}$ represents the transmission factor through the overall thickness $t_{n}$ of the neck phantom $(10.81 \pm 0.01 \mathrm{~cm})$ with an overall effective linear attenuation coefficient $\mu_{e}$ (Siegel et al 1999). In order to perform the activity quantification in a realistic way, the transmission factor value was estimated by using the theoretical attenuation coefficients of adipose tissue and spine at $364 \mathrm{keV}$ $\left(0.106 \mathrm{~cm}^{-1}\right.$ and $0.152 \mathrm{~cm}^{-1}$, respectively (Hubbell and Seltzer 2004)) together with standard spine dimensions reported by the ICRP (Siegel et al 1999). This method leads to a $e^{\mu_{e}}$ value different from only $0.65 \%$ compared to that calculated with the actual phantom geometry and material attenuation coefficients. $C$ represents the system calibration factor extrapolated from the distance-sensitivity curve without absorbing medium, as described above. The factor $f_{j}$ represents the source self-attenuation correction, estimated from the source region attenuation coefficient $\mu_{j}$ and the source thickness $t_{j}$ as:

$$
f_{j}=\frac{\mu_{j} t_{j} / 2}{\sinh \left(\mu_{j} t_{j} / 2\right)} .
$$

The factor $f_{j}$ involves only the thyroid region characteristics $\left(0.106 \mathrm{~cm}^{-1}\right.$ at $364 \mathrm{keV}$ (Hubbell and Seltzer 2004)) and will not deviate significantly from 1 until $t_{j}$ become large. The thicknesses of the lobes $t_{j}$ were estimated from the phantoms lateral images as previously described.

The accuracy of the quantification achieved with the mobile camera is estimated from the recovery coefficient (RC), corresponding to the ratio between the measured activity $A_{j}$ and the known activity in the thyroid phantom corrected for its decay over time.

\section{Results}

\subsection{Intrinsic performance of the camera}

In order to qualitatively compare the relative response of the two tested $\mathrm{CeBr}_{3}$ in terms of light yield and light collection, the non-calibrated pulse height energy spectra corresponding to a central $4 \mathrm{~mm}$ collimated ${ }^{133} \mathrm{Ba}$ source are shown in figure 6 (left). The higher peak positions measured with the $6 \mathrm{~mm}$ thick scintillator suggest a slight decrease in the light collection as the thickness of the crystal increases, which may be due both to the self-absorption of the scintillation light and to variations in the quality of the scintillating material. The calibrated ${ }^{133} \mathrm{Ba}$ spectra show the principal emission peaks at (a) $356 \mathrm{keV}$ (the $383 \mathrm{keV}$ is not resolved), (b) $302 \mathrm{keV}$ and (c) $276 \mathrm{keV}$, (d) the $356 \mathrm{keV}$ Compton edge at $207 \mathrm{keV}$, (e) the backscatter peak at $149 \mathrm{keV}$ and (f) the superimposition of the ${ }^{133} \mathrm{Ba}$ emission at $81 \mathrm{keV}$ and the characteristics tungsten $\mathrm{x}$-rays (figure 6 (right)). The mean energy resolution (ER) measured in the UFOV, CFOV and $\overline{\text { CFOV }}$ together with the corrected overall ER are summarized in table 1 . The energy resolution and intensity maps on the whole field of view of the camera are shown in figure 7. Non-significant differences are observed between the two scintillators for what concern their average and overall ER. For both scintillators, the use of reflective coatings on the edges allows to obtain a fairly uniform energy response, visible from the small ER standard deviation in the UFOV and from the energy map (figures 7(a), (d)). This is correlated with the almost constant light collection over the whole field of view (figures 7(b), (e)). The correction of the spatial variations in the light collection mainly impacts the overall position and width of the full-energy peak near the edges of the crystal and results in a significant improvement of the overall ER relative to the mean ER in the UFOV for both samples (below $8 \%$ at $356 \mathrm{keV}$ ) (table 1). Without any correction of the energy response uniformity, the overall ER measured from a flood field irradiation is $11.17 \pm 0.05 \%$ for the $6 \mathrm{~mm}$ thick $\mathrm{CeBr}_{3}$ scintillator.

The flood field images obtained with the ${ }^{133} \mathrm{Ba}$ source are shown in figures 7 (c), (f). The corresponding IU and DU values are summarized in table 2. The IU and DU values measured in the CFOV are less than $3 \%$ and are 

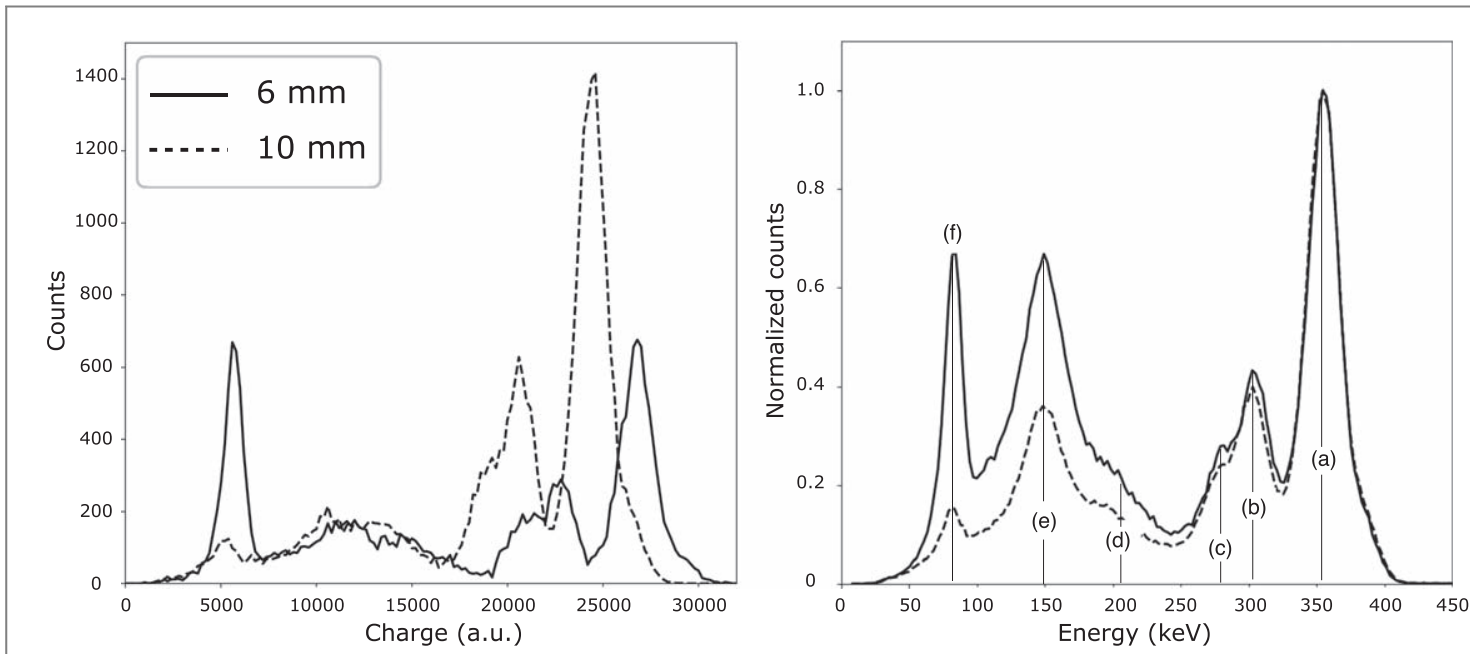

Figure 6. Non-calibrated (left) and calibrated (right) pulse height energy spectra measured with the $6 \mathrm{~mm} \mathrm{and} 10 \mathrm{~mm}^{\mathrm{mick}} \mathrm{CeBr}_{3}$ scintillators and a $4 \mathrm{~mm}$ diameter collimated ${ }^{133} \mathrm{Ba}$ source.
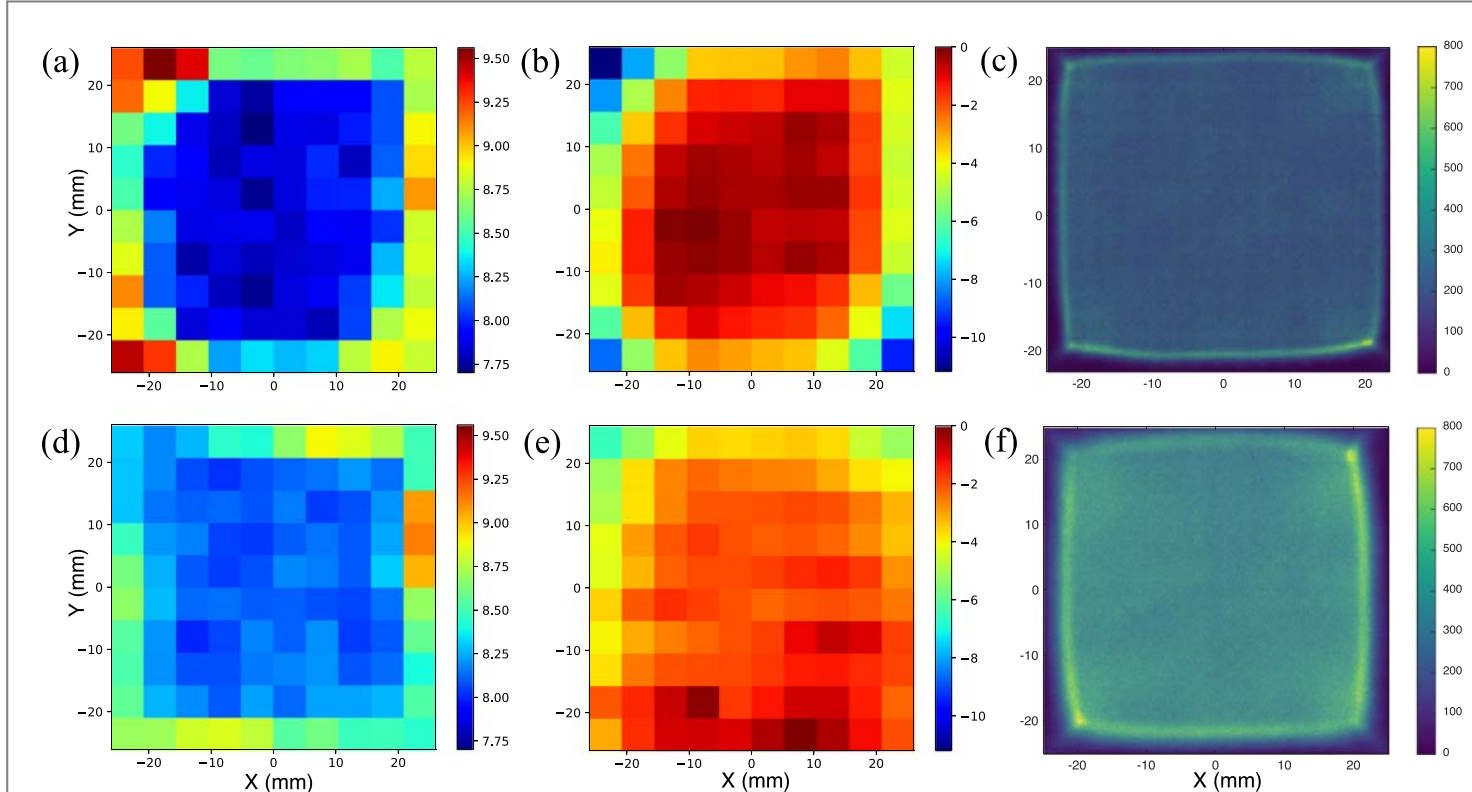

Figure 7. (a), (d) Energy resolution (FWHM), (b), (e) relative light collection maps and (c), (f) flood field images (counts) measured with the $6 \mathrm{~mm}$ (top) and $10 \mathrm{~mm}$ (bottom) thick $\mathrm{CeBr}_{3}$ scintillators and $\mathrm{a}^{133} \mathrm{Ba}$ source. The relative light loss is measured in relation to the position corresponding to the highest light collection.

Table 1. Mean value of the FWHM energy resolution (\%) in the UFOV, CFOV and $\overline{\mathrm{CFOV}}$ of the camera at $356 \mathrm{keV}\left({ }^{133} \mathrm{Ba}\right)$, together with their overall ER measured after correction of the energy response uniformity. The uncertainties correspond to the standard deviation of the energy resolution values over the detector's field of view.

\begin{tabular}{lcccc}
\hline Thickness & UFOV & CFOV & $\overline{\text { CFOV }}$ & Overall \\
\hline $6 \mathrm{~mm}$ & $8.14 \pm 0.03$ & $7.86 \pm 0.03$ & $8.67 \pm 0.03$ & $7.68 \pm 0.01$ \\
$10 \mathrm{~mm}$ & $8.12 \pm 0.02$ & $7.96 \pm 0.01$ & $8.41 \pm 0.02$ & $7.84 \pm 0.01$ \\
\hline
\end{tabular}

close to typical values measured during quality controls of commercial gamma cameras (O'Connor and Vermeersch 1991, Cherry et al 2012). The significant degradation of IU in the UFOV is assumed to be related to the spatial compression of the image near the reflective edges, which causes an artificial accumulation of the measured activity. The better DU achieved with the $10 \mathrm{~mm}$ thick scintillator is obviously due to the broader light distribution reaching the photodetection system. This reduces the positioning errors due to the discrete nature 
Table 2. Integral uniformity (IU) and

differential uniformity (DU) in the UFOV and

CFOV of the camera at $356 \mathrm{keV}\left({ }^{133} \mathrm{Ba}\right)$ after

correction of the energy response uniformity and for two scintillator thicknesses.

\begin{tabular}{llcc}
\hline & & $6 \mathrm{~mm}$ & $10 \mathrm{~mm}$ \\
\hline IU (\%) & UFOV & 11.53 & 17.89 \\
& CFOV & 2.41 & 2.97 \\
DU (\%) & UFOV & 2.82 & 4.96 \\
& CFOV & 1.26 & 0.97 \\
\hline
\end{tabular}

Table 3. Intrinsic spatial resolution (SR), local intrinsic spatial distortion (LISD) and absolute linearity (AL) in the UFOV $\mathrm{CFOV}$ and $\overline{\mathrm{CFOV}}$ of the camera at $356 \mathrm{keV}\left({ }^{133} \mathrm{Ba}\right)$.

\begin{tabular}{lccc}
\hline & & $6 \mathrm{~mm}$ & $10 \mathrm{~mm}$ \\
\hline \multirow{3}{*}{$\mathrm{SR}(\mathrm{mm})$} & $\mathrm{UFOV}$ & $0.74 \pm 0.28$ & $1.53 \pm 1.08$ \\
& $\mathrm{CFOV}$ & $0.61 \pm 0.04$ & $1.05 \pm 0.10$ \\
& $\overline{\mathrm{CFOV}}$ & $0.91 \pm 0.35$ & $2.20 \pm 1.34$ \\
$\mathrm{LISD}(\mathrm{mm})$ & $\mathrm{UFOV}$ & $0.15 \pm 0.10$ & $0.22 \pm 0.11$ \\
& $\mathrm{CFOV}$ & $0.06 \pm 0.03$ & $0.08 \pm 0.05$ \\
$\mathrm{AL}(\mathrm{mm})$ & $\mathrm{CFOV}$ & $0.20 \pm 0.13$ & $0.41 \pm 0.13$ \\
& UFOV & 2.02 & 3.03 \\
& CFOV & 0.3 & 0.50 \\
\hline
\end{tabular}

of the photodetector, which leads to the slight appearance of the detector array pattern in the flood images measured with the $6 \mathrm{~mm}$ thick scintillator (figure $7(\mathrm{c})$ ). Conversely, this broader light distribution is also more sensitive to distortions due to reflective edges, resulting in greater degradation of uniformity in the UFOV with the $10 \mathrm{~mm}$ thick scintillator. The small difference observed between IU and DU demonstrates that there is no systematic spatial variation across the field of view of the camera.

The intrinsic spatial resolution (SR), local intrinsic spatial distortion (LISD) and absolute linearity (AL) are summarized in table 3 . The narrower light distribution produced at the output of the $6 \mathrm{~mm}$ thick scintillator compared to that obtained with the $10 \mathrm{~mm}$ sample allows to obtain a sub-millimeter intrinsic spatial resolution at $356 \mathrm{keV}$ in the whole field of view $(0.74 \pm 0.28 \mathrm{~mm}$ in the UFOV) (figures $8(\mathrm{c})$, (d)). The improvement observed for the $6 \mathrm{~mm}$ thick crystal is more significant close to the edges thanks to better spatial linearity (figures $8(\mathrm{~g})$, (h)). If the spatial resolution is degraded with the $10 \mathrm{~mm}$ thick scintillator, it remains very high at $356 \mathrm{keV}$, as can be seen qualitatively in the $17 \times 17$ scanning images (figures $8(\mathrm{a}),(\mathrm{b}))$ and very close to one millimeter in the CFOV (table 3 and figures 8 (c), (d)). Both scintillators have a comparable and very low LISD in the CFOV, which underlines the very good ability of the reconstruction algorithm to identify the center of the light distribution independently of its spread on the photodetector (table 3 and figures $8(\mathrm{e})-(\mathrm{h})$ ). However, the influence of the reflective coatings on the edges is clearly highlighted by the spatial compression on the scan images (figures 8(a), (b)) and the distortion maps (figures 8(e), (f)). The light distribution can no longer be modeled by a Scrimger-Becker function on the periphery of the camera, which translates in a poorer reconstruction of the image. This distortion of the light distribution is even stronger for the $10 \mathrm{~mm}$ thick sample because the wider light distribution increases the reflections at the edges. More quantitatively, the absolute linearity in the UFOV increases from $2 \mathrm{~mm}$ to $3 \mathrm{~mm}$ for the $6 \mathrm{~mm}$ and $10 \mathrm{~mm}$ thick scintillators respectively, which corresponds to the maximum distortion observed in the corners of the image.

In light of these results, one can notice that the $6 \mathrm{~mm}$ thick $\mathrm{CeBr}_{3}$ offers high $\mathrm{ER}(7.68 \pm 0.01 \%)$, submillimetric SR $(0.74 \pm 0.28 \mathrm{~mm})$ and very low spatial distortion $(0.15 \pm 0.10 \mathrm{~mm})$ thanks to its high light yield and light collection. The high spectrometric performance achieved with the $10 \mathrm{~mm}$ thick reflecting $\mathrm{CeBr}_{3}$ also makes it a good potential candidate that can offer excellent pulse height uniformity and, more importantly, an increase of about $40 \%$ in photopeak efficiency compared to the $6 \mathrm{~mm}$ thick scintillator, which could help to reduce the uncertainties on dose quantification. However, it is first necessary to improve spatial performance close to the reflective edges, which reduces the effective useful FOV due to compression effects. For these reasons, the $6 \mathrm{~mm}$ thick $\mathrm{CeBr}_{3}$ has been chosen as the scintillator to be integrated in the first $5 \times 5 \mathrm{~cm}^{2}$ prototype of the mobile gamma camera. 


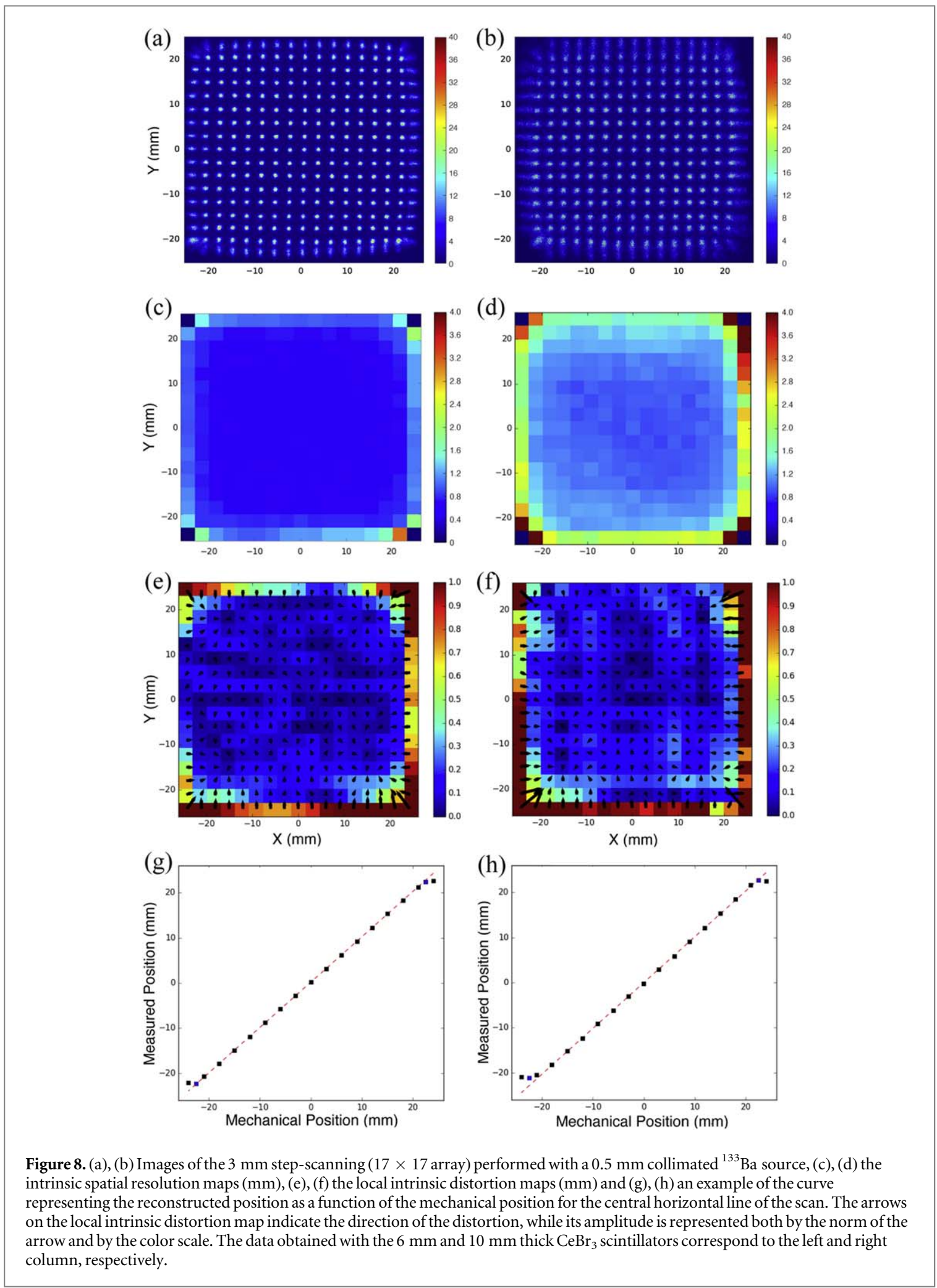

\subsection{Camera calibration}

The spatial resolution of the system varies linearly as a function of the source distance from a minimum value of $2.21 \pm 0.03 \mathrm{~mm}$ at $1 \mathrm{~cm}$ from the collimator to a maximum of $5.47 \pm 0.05 \mathrm{~mm}$ at $15 \mathrm{~cm}$. A value of $3.14 \pm 0.03 \mathrm{~mm}$ is measured at $5 \mathrm{~cm}$, to be compared to the $2.5 \mathrm{~mm}$ FWHM resolution estimated from Monte Carlo simulations with a line source. Figure 9 shows the sensitivity of the mobile camera as a function of the diameter and the distance of the source from the collimator without an absorbing medium. The sensitivity was measured without and with TEW correction (figure 9 (left) and figure 9 (middle)). The sensitivity decreases with the distance for all sources. This trend is in contradiction with the geometrical behavior of parallel hole collimators (distance-independent sensitivity (Siegel et al 1999)), but is in agreement with other results found in 

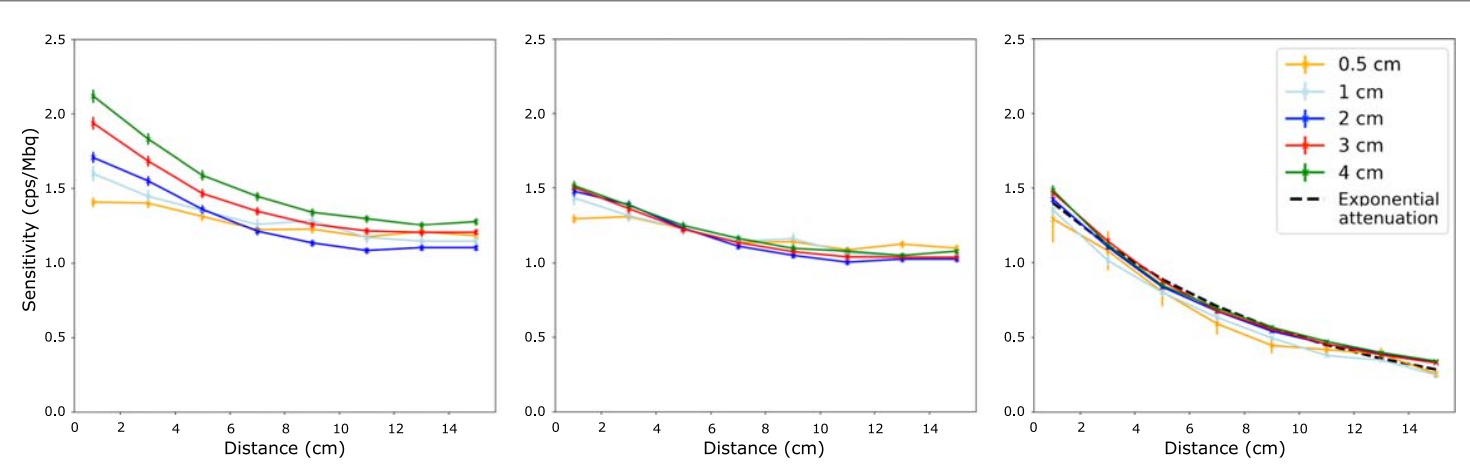

Figure 9. Sensitivity of the camera as a function of the diameter and the distance of the source from the collimator without (left and middle) and with absorbing medium (right). The sensitivity is measured without (left) and with TEW correction (middle and right).
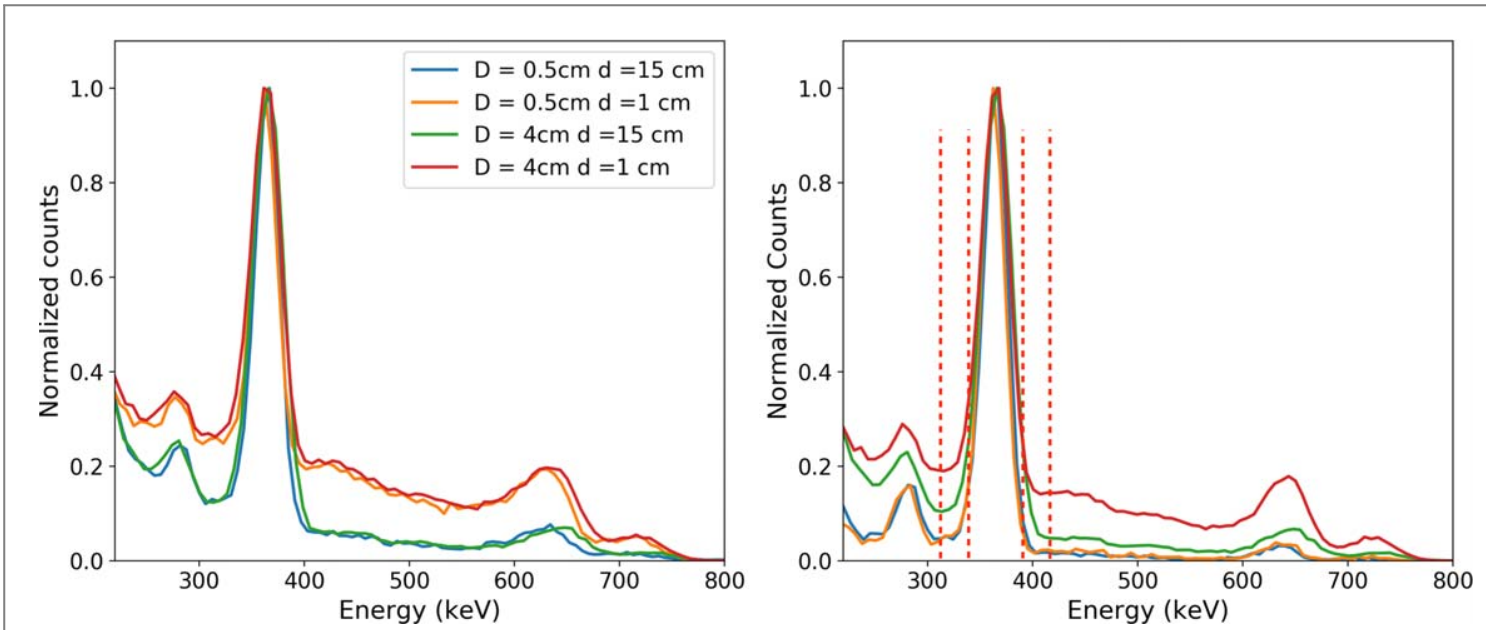

Figure 10. Normalized spectra measured with the $0.5 \mathrm{~cm}$ and $5 \mathrm{~cm}$ diameter cylindrical ${ }^{131}$ I sources at $1 \mathrm{~cm}$ and $15 \mathrm{~cm}$ from the collimator. Events detected in the whole FOV (left) and within a ROI on the source image (right) were considered. The three energy windows used for event selection in the $364 \mathrm{keV}$ photopeak of ${ }^{131} \mathrm{I}$ and for TEW correction are shown.

the literature (Barquero et al 2017). It can be understood by observing the ${ }^{131}$ I normalized spectra measured with the smallest and largest cylindrical sources at $1 \mathrm{~cm}$ and $15 \mathrm{~cm}$ from the collimator for events detected in the whole FOV (figure 10 (left)) or within the circular ROIs on the source image obtained from the threshold method (figure 10 (right)). Despite the optimization of the high energy collimator, its sensitivity is still influenced by the scattering and penetration of high-energy gamma rays, which increase at short distances and for large diameter sources. Since scatter events are distributed over the entire FOV of the camera, the ROI selection removes a large part of this contribution for small sources, but it remains important for large sources especially at short distances (figure 10 (right)). As a consequence, the $0.5 \mathrm{~cm}$ diameter source is the only one that shows a nearly complete independence of the amount of scatter and penetration with distance regardless of the use of the TEW correction and thus, an almost constant sensitivity. Because the contribution of scatter is higher for large sources and at short distances, the impact of the TEW correction is more significant for these configurations (figure 9 (middle)). Combined with the threshold method for the ROI definition, which allows a strong reduction of the partial volume effect for source sizes close to the spatial resolution of the camera, the TEW correction makes the sensitivity very little dependent on the source dimensions with a mean value of $1.23 \pm 0.01 \mathrm{cps} / \mathrm{MBq}$ at a distance of $5 \mathrm{~cm}$, close to the $1.33 \pm 0.02 \mathrm{cps} / \mathrm{MBq}$ value estimated from Monte Carlo simulation. Finally, figure 9 (right) shows the sensitivity measured with absorbing medium with TEW correction. The decrease in sensitivity with distance is well described by the theoretical attenuation of the absorbing medium $\left(\mu=0.111 \mathrm{~cm}^{-1}\right)$. The calibration factors $C$ and $C_{a b s}$ used for the quantification with singleview planar images were extrapolated from these distance-sensitivity curves.

\subsection{Preclinical planar imaging using homogenous 3D-printed phantoms}

The high imaging ability of our device is first illustrated in figure 11, which compares the images of the two healthy thyroid phantoms obtained with the mobile camera (left) and with a standard gamma camera equipped 

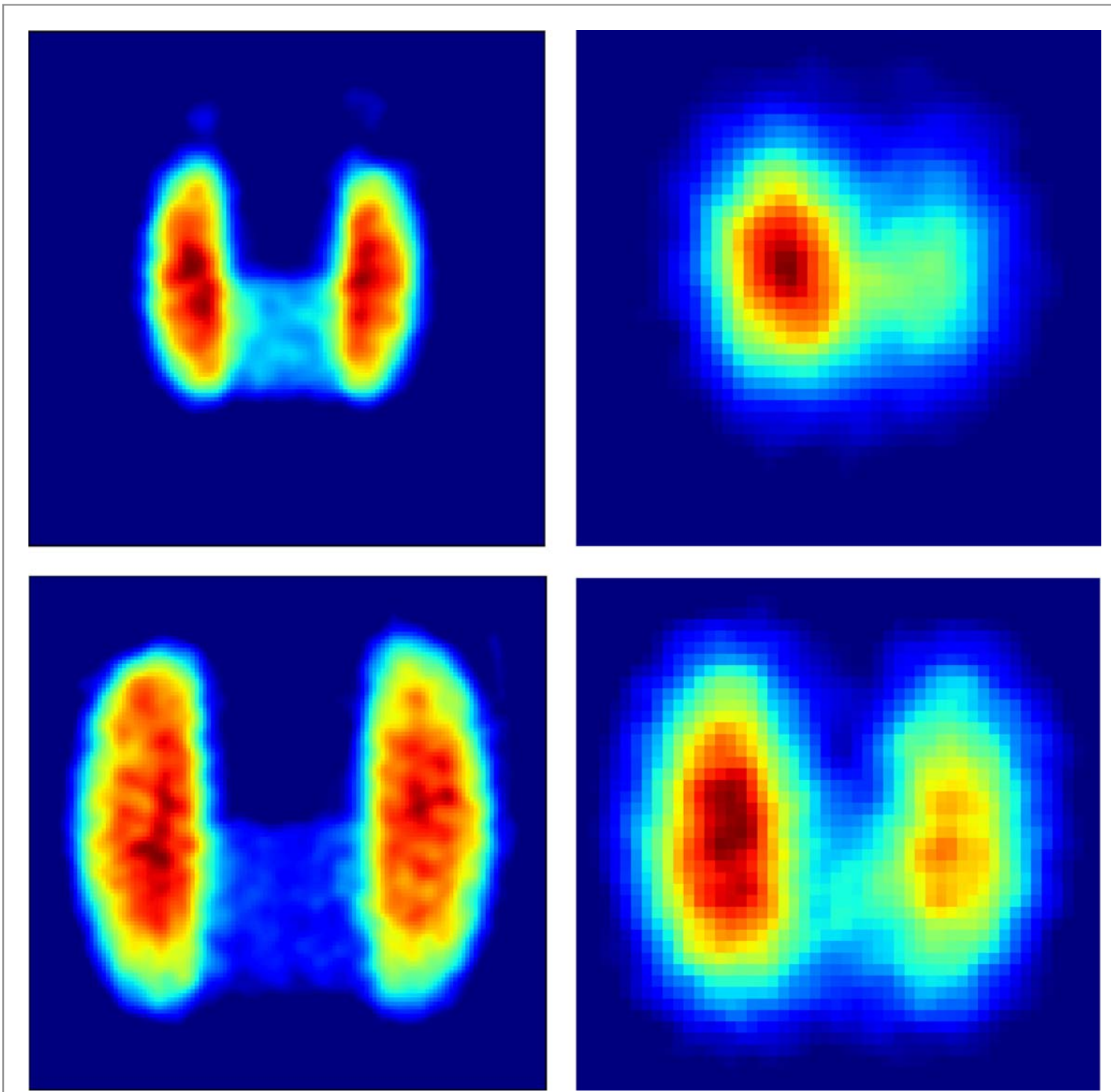

Figure 11. Images of the FANTHY5y (top) and FANTHY15y (bottom) thyroid phantoms obtained with the mobile gamma camera (left) and with a clinical gamma camera equipped with a high-energy collimator (Siemens Symbia T2 GC) placed at a minimum distance of $10 \mathrm{~cm}$ from phantoms (right). The $X$ and $Y$ scales are the same on all the images.

with a high-energy collimator (Siemens Symbia T2 GC, spatial resolution of $13.4 \mathrm{~mm}$ at $10 \mathrm{~cm}$ ). One can qualitatively notice how the optimized characteristics of the mobile camera (high spatial resolution, reduced scattering and penetration in the collimator and compactness, which allows it to be placed very close to the structure of interest) make it possible to greatly improve the quality of the image.

\subsubsection{Thickness and depth estimation}

The ability of the camera to measure the thickness and depth of the structures of interest is summarized in table 4 and is compared to the actual geometric values of the phantoms. The measured thicknesses of the lobes are in good agreement with the expected one (relative errors of 7.3\% and 3.7\% for FANTHY5y and FANTHY15y, respectively). Thanks to its compactness, the camera can always be positioned as close as possible to the structure of interest in order to estimate their dimension accurately. However, it can be noted that a small error on the lobe thickness has a small impact on the quantification under the conditions of our study. Indeed, the lobe thickness $t_{j}$ comes into play when calculating the factor $f_{j}$ (equation (3)), which deviates of less than $0.2 \%$ from the unity for the greatest thickness found and for the thyroid attenuation coefficient. For what concerns the depth values, the relative errors measured for the FANTHY5y and FANTHY15y phantoms ( $8.5 \%$ and $8.3 \%$, respectively) could be related to slight misalignment of the camera with respect to the neck (in the order of one millimeter). As introduced previously, the estimation of the lobes depth in the neck is important because it allows to extrapolated the calibration factor from the sensitivity-distance curves. The small inaccuracy in depth estimation such as the one reported results in a variation of the calibration factor of less than $0.4 \%$ for both 


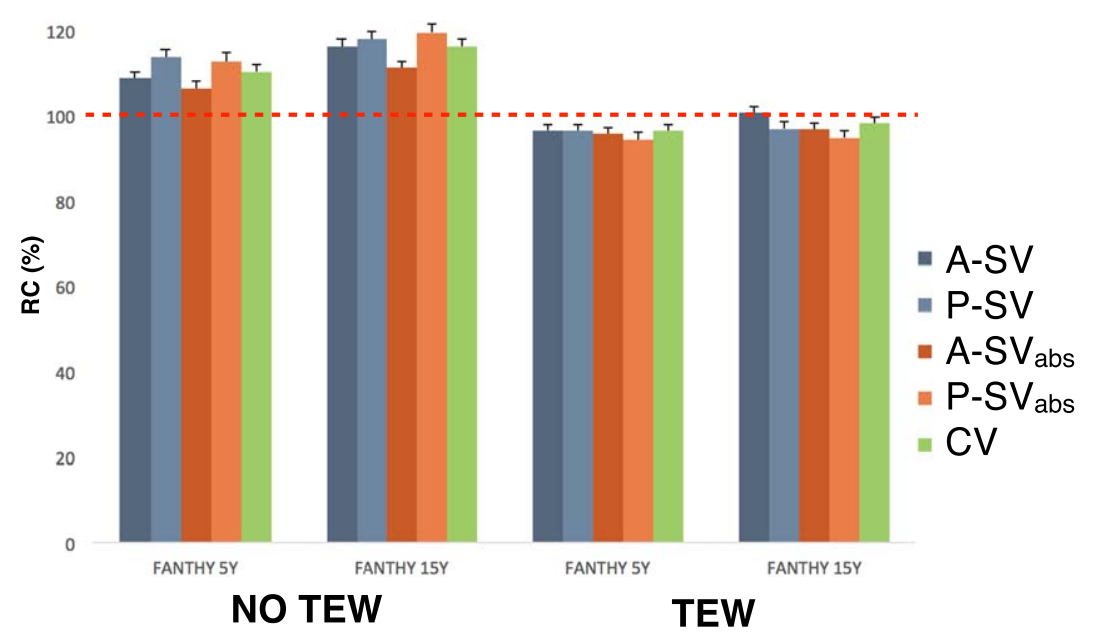

Figure 12. Recovery coefficients (RC) measured for the thyroid phantoms activity.

Table 4. Dimensions and depths of the thyroid lobes measured from the lateral images. Comparison with the actual geometric values.

\begin{tabular}{lccccc}
\hline & \multicolumn{2}{c}{ Thickness $(\mathrm{mm})$} & & \multicolumn{2}{c}{ Depth $(\mathrm{mm})$} \\
\cline { 2 - 3 } \cline { 5 - 6 } & Expected & Measured & & Expected & Measured \\
\hline FANTHY5y & 7.63 & $8.23 \pm 0.61$ & & 11.8 & $12.9 \pm 0.82$ \\
FANTHY15y & 18.6 & $19.32 \pm 0.48$ & & 15.4 & $16.8 \pm 0.90$ \\
\hline
\end{tabular}

Table 5. Recovery coefficients (RC) measured for the thyroid phantoms activity using two single views (SV) methods with the anterior (A) and posterior (P) images and the conjugate view approach $(\mathrm{CV})$. The calibration factor was measured with $\left(\mathrm{SV}_{a b s}\right)$ and without an absorbing medium (SV). All methods were investigated with and without TEW correction.

\begin{tabular}{lccccc}
\hline & \multicolumn{2}{c}{ NO TEW } & & \multicolumn{2}{c}{ TEW } \\
\cline { 2 - 3 } \cline { 5 - 6 } RC $(\%)$ & FANTHY 5y & FANTHY 15y & & FANTHY 5y & FANTHY 15y \\
\hline A-SV & $108.62 \pm 1.67$ & $116.08 \pm 1.77$ & & $96.45 \pm 1.50$ & $100.53 \pm 1.55$ \\
P-SV & $113.57 \pm 1.75$ & $117.85 \pm 1.81$ & & $96.44 \pm 1.52$ & $96.88 \pm 1.51$ \\
A-SV $_{a b s}$ & $106.29 \pm 1.63$ & $111.03 \pm 1.70$ & & $95.65 \pm 1.43$ & $96.74 \pm 1.51$ \\
P-SV & $112.63 \pm 2.01$ & $119.45 \pm 2.05$ & & $94.12 \pm 1.79$ & $94.75 \pm 1.73$ \\
CV & $110.25 \pm 1.72$ & $116.2 \pm 1.79$ & & $96.23 \pm 1.53$ & $98.04 \pm 1.54$ \\
\hline
\end{tabular}

phantoms (estimated from the sensitivity curve of the $2 \mathrm{~cm}$ and $3 \mathrm{~cm}$ diameter cylinder reported in figure 9). The measured values of the thickness and depth of the lobes have been used for the activity quantification.

\subsubsection{Preliminary assessment of the camera ability to quantify ${ }^{131}$ I with homogeneous activity distribution}

The results of the activity quantification study for the two thyroid phantoms are summarized in table 5 and figure 12. An almost perfect activity quantification is achieved with FANTHY15y when using the single view method with TEW correction. The recovery coefficient is almost $100 \%$ with the anterior image and $97 \%$ with the posterior view. The recovery factor measured with FANTHY5y is around $96 \%$ for both views. No particular trend can be highlighted between all different quantification methods for the small thyroid phantom. The lowest recovery coefficient measured with TEW correction (around 94\%) is obtained when using the posterior single view method with attenuation. This effect is due to the use of a calibration factor extrapolated from the sensitivity-distance curve with attenuation, which was obtained using an absorbing medium with a slightly different attenuation coefficient compared to the one of the neck phantom $\left(0.111 \mathrm{~cm}^{-1}\right.$ for the absorbing medium used during the calibration procedure and $0.116 \mathrm{~cm}^{-1}$ for the phantom). The underestimation of attenuation leads to an underestimation of the activity, which is more important with the posterior view since it corresponds to a greater length of attenuation. The single view method based on the calibration factor measured 
without an absorbing medium and a post-correction of the attenuation results in better quantification because it uses the actual attenuation of the absorbing medium. The conjugate view protocol, in which both anterior and posterior views are used, allows obtaining a RC value of $96 \%$ and $98 \%$ for FANTHY5y and FANTHY15y, respectively. The activities inside the phantoms measured without TEW correction are overestimated by an average of $9.8 \%$ and $15.8 \%$ for FANTHY5y and FANTHY15y, respectively. This shows that the amount of scattering measured with the thyroid phantoms is higher than during the calibration with the cylindrical sources, due to a larger scattering volume.

\section{Discussion and conclusion}

We presented the design, characterization and calibration of the first prototype of a high-resolution mobile gamma camera with a $5 \times 5 \mathrm{~cm}^{2}$ FOV specifically optimized to improve the quantitative assessment of the distribution and biokinetics of ${ }^{131} \mathrm{I}$ after treatment administration. Compared to standard large field of view cameras equipped with high-energy collimators, the image quality achieved with our device is significantly improved thanks to its submillimetric intrinsic spatial resolution and reduced penetration and scattering in the optimized tungsten collimator (figure 11). However, these performances cannot be rigorously compared to those of existing technologies, because our experimental protocol presents slight divergences from the NEMA standards, in particular to take into account the specificities of miniaturized cameras (Bhatia et al 2015). The system calibration leads to an overall energy resolution of $7.68 \pm 0.01 \%$, a spatial resolution of $3.14 \pm 0.03 \mathrm{~mm}$ and a sensitivity of $1.23 \pm 0.01 \mathrm{cps} / \mathrm{MBq}$ at a distance of $5 \mathrm{~cm}$. This performance is consistent with the initial objectives and clinical requirements described in the Introduction of this work. Moreover, the compactness and mobility of our camera should allow improving image resolution by reducing the detector-targeted structures distance (thyroid, tumor remnants, metastatic nodes or salivary glands) and optimizing their detectability by adjusting the angular view.

Preliminary results reported for two different 3D-printed thyroid phantoms filled with ${ }^{131}$ I demonstrate that these performance and features are well suited to achieve a high quantification capability in a fairly realistic clinical context. Recovery factors for the thyroid activity deviate by less that $3 \%$ from unity, even using a single view method combined with a simple TEW correction but without partial volume effect correction. Indeed, the high spatial resolution of the mobile camera reduces the partial volume effect, by allowing a more accurate selection of accumulated activity within ROIs using threshold methods. This high spatial performance and the ability of the camera to be placed close to the thyroid and to adjust the angular view also gives access to precise anatomical information (position and dimensions of structures), which are then used to improve quantification by correcting attenuation, source self-absorption or activity superimposition. Considering the average value of the activity administrated to the patient during hyperthyroidism treatments (between 5 and $20 \mathrm{mCi}$ ) and the high thyroid uptake (mean value ranging between 40 and 50\% of the administrated activity) (Stokkel et al 2010), the same accuracy of the quantification process could be attained with image acquisitions of few $\min (5-10 \mathrm{~min})$. This should facilitate the acquisition of multiple images over time to estimate cumulative activity. Because the sensitivity of the camera decreases with distance and is also slightly dependent on the size of the source due to the influence of high-energy gamma scattering within the collimator, this preliminary study also emphasizes the importance of accurate calibration for patient-specific quantification of absorbed doses (Barquero et al 2017). The accuracy of ${ }^{131}$ I thyroidal activity quantification achieved with our mobile camera will be compared to that obtained with current techniques based on planar gamma cameras and/or SPECT/CT in a more comprehensive study performed with the clinical prototype of the mobile camera currently under development.

However, several experimental biases still limit the extrapolation of these very good results to a full reliability of the quantification process and to the possible clinical usefulness of the mobile camera. In particular, the quantification of the activity needs to be evaluated for small heterogeneities modeling nodules or tumor remnants by using 3D-printed thyroid phantoms with spherical inserts embedded or partially embedded in the thyroid and different uptake ratios compared to normal tissues. Due to the massive amount of activity administered in clinical practice, high-energy primary radiation or scattering background noise coming from the extrathyroidal area of radiotracer uptake (e.g. bladder, stomach, adipose tissue), which could bypass the collimator, could also be a major issue. Their influence must be considered since they could strongly contribute to camera dead time and image contrast degradation. Consequently, the shielding around the scintillator used for the clinical prototype of the mobile camera will be optimized with Monte Carlo simulations (nature of the absorbing material, thickness and positioning of the shielding) in order to strongly reduce radiation penetration from the sides and back of the camera while maintaining its compactness and mobility. The next instrumental step toward a fully operational camera in the clinic is to implement a $10 \times 10 \mathrm{~cm}^{2}$ field of view detector suited to the size of the thyroid gland even when enlargement occurs, such as in the case of Graves' disease. The second objective is to take advantage of the camera's imaging capabilities during treatment planning, which uses low 
levels of tracer activity. To improve the versatility of the camera performance, two interchangeable detection heads will therefore be developed: a high-sensitivity configuration for imaging during treatment planning of hyperthyroidism or imaging of metastatic lymph nodes and salivary glands during treatment of thyroid cancers (overall spatial resolution around $7 \mathrm{~mm}$ and sensitivity of about $25 \mathrm{cps} / \mathrm{MBq}$ at a distance of $5 \mathrm{~cm}$ ) and a high resolution (and low-sensitivity) configuration similar to the one used with the feasibility prototype for estimating the absorbed dose in thyroid or tumor remnants after treatment administration (overall spatial resolution around $3 \mathrm{~mm}$ and sensitivity around $4 \mathrm{cps} / \mathrm{MBq}$ ). Compared to the current configuration $(1.23 \pm 0.01 \mathrm{cps} / \mathrm{MBq})$, an improvement in sensitivity will be first obtained by adjusting the geometric features of the high resolution collimator to increase its efficiency by a factor of 2.5 while slightly degrading its spatial resolution (from 2 to $3 \mathrm{~mm}$ for a source distance of $5 \mathrm{~cm}$ ). Second, by increasing the thickness of the $\mathrm{CeBr}_{3}$ scintillator from $6 \mathrm{~mm}$ to $10 \mathrm{~mm}$, allowing a gain of about $50 \%$ at $364 \mathrm{keV}$ without degrading the energy response and with only a slight deterioration in spatial performance, as shown in our study (tables 1 and 3 ). However, more sophisticated reconstruction algorithms of the interaction point, such as artificial neural networks, should be implemented in order to avoid spatial distortion near the edges and maintain a fully usable field of view (Iborra et al 2019). The global gain in sensitivity will allow reducing the acquisition time of each image to a maximum of 10 min even under the least favorable activity conditions (detection of tumor remnants with less than $1 \%$ uptake after administration of $100 \mathrm{mCi}$ of $\left.{ }^{131} \mathrm{I}\right)$. The intrinsic properties of the tungsten collimators could also be further improved by optimizing the printing parameters. Indeed, the selective melting method chosen for the manufacturing of the collimator and which consists in the fusion of grains of tungsten powders with a high-power laser, implies a certain porosity of the material and, therefore, a lower effective density than pure tungsten, which can range from 80 to $96 \%$ of the theoretical density $\left(19.3 \mathrm{~g} \mathrm{~cm}^{-3}\right)$ depending on the printing process (Gear et al 2019). The finite size of the laser beam and tungsten powder grains also implies an inaccuracy in the actual size of the holes and septa obtained, which can modify the characteristics of the collimator compared to those expected (figure 1 (right)), as illustrated by the differences between the experimental and simulated data for spatial resolution and sensitivity. Increased density and better accuracy of geometric features can be obtained by tuning the printing parameters, in terms of scan speed and powder diameter. Beyond the increased field of view, it is also necessary to significantly improve the counting capabilities of the photodetection system. Indeed, the camera is currently limited by an acquisition rate of $5 \mathrm{kcps}$. New readout electronics with a faster communication protocol will be implemented to achieve a maximum counting capacity of about $200 \mathrm{kcps}$, opening the door to early imaging just after treatment is administered.

\section{Acknowledgments}

This work was supported by IN2P3. The authors thank A Forbes and L Ammour from the Nuclear Medicine department of Hôpital Bicètre, for their help with the evaluation of the mobile camera on the radioactive phantoms. They also thank Olivier Caselles from the medical physics department of the Claudius Regaud Institute who provided the images of the thyroid phantoms acquired with the standard gamma camera.

\section{ORCID iDs}

Carlotta Trigila (1D https://orcid.org/0000-0002-0414-9369

Marc-Antoine Verdier (iD https://orcid.org/0000-0002-1375-2215

Laurent Ménard (10 https://orcid.org/0000-0001-7867-1881

\section{References}

Association, National Electrical Manufacturers 2018 NEMA Standards publication NU 1-2018, Performance measurements of gamma cameras. In: Rosslyn, VA.

Barquero Ret al 2017 131I activity quantification of gamma camera planar images Phys. Med. Biol. 62 909-66

Beaumont T et al 2017 Development and test of sets of 3D printed agespecific thyroid phantoms for 131I measurements Phys. Med. Biol. 62 4673-93

Bhatia B S et al 2015 A scheme for assessing the performance characteristics of small field-of-view gamma cameras Phys. Med. 31 98-103

Cherry S R, Sorenson J A and Phelps M E 2012 Physics in Nuclear Medicine e-Book (Elsevier Health Sciences)

Dewaraja Y, Li J and Koral K 1998 Quantitative 131I SPECT with triple energy window compton scatter correction IEEE Trans. Nucl. Sci. 45 3109-14

Dewaraja Y K et al 2012 MIRD pamphlet No. 23: quantitative SPECt for patient-specific 3-dimensional dosimetry in internal radionuclide therapy J. Nucl. Med. 53 1310-25

Dewaraja Y K et al 2013 MIRD pamphlet No. 24: guidelines for quantitative 131i spect in dosimetry applications J. Nucl. Med. 54 2182-8

Dinu N et al 2015 SiPM arrays and miniaturized readout electronics for compact gamma camera nuclear instruments and methods in physics research section a: accelerators, spectrometers Detectors Assoc. Equip. 787 367-72 
Eary J F et al 1997 Importance of pre-treatment radiation absorbed dose estimation for radioimmunotherapy of non-hodgkin's Lymphoma Nuclear Medicine and Biology 24 635-8

Eberlein U, Cremonesi M and Lassmann M 2017 Individualized dosimetry for theranostics: necessary, nice to have, or counterproductive? J. Nucl. Med. 58 97S-103S

European Council Directive 2013 Council Directive 2013/59/Euratom of 5 December 2013 laying down basic safety standards for protection against the dangers arising from exposure to ionising radiation, and repealing Directives 89/618/Euratom, 90/641/ Euratom, 96/29/Euratom, 97/43/Euratom and 2003/122/Euratom. en. In: European Commission, p. 73.

Eyben F E, Baumann G S and Baum R P 2018 PSMA diagnostics and treatments of prostate cancer become mature Clin. Transl. Imaging 6 $145-8$

Flux G D et al 2018 From fixed activities to personalized treatments in radionuclide therapy: lost in translation? Eur. J. Nucl. Med. Mol. Imaging 45 152-4

Flux Get al 2006 The impact of PET and SPECT on dosimetry for targeted radionuclide therapy Z. Med. Phys. 1647-59

Garcia H P and Barquero R 2017 The HURRA filter: an easy method to eliminate collimator artifacts in high-energy gamma camera images Revista Española Med. Nucl. Imagen Mol. 3627-36

Garin E et al 2017 High impact of macroaggregated albumin-based tumour dose on response and overall survival in hepatocellular carcinoma patients treated with 90Y-loaded glass microsphere radioembolization Liver Int. 37 101-10

Gear J I, Taprogge J, White O and Flux G D 2019 Characterisation of the attenuation properties of 3Dprinted tungsten for use in gamma camera collimation EJNMMI Phys. 6 1-17

Iborra A et al 2019 Ensemble of neural networks for 3D position estimation in monolithic PET detectors Phys. Med. Biol. 64 195010

Jan S et al 2004 GATE: a simulation toolkit for PET and SPECT Phys. Med. Biol. 49 4543-61

Kesner A L and Bodei L 2018 modern radiopharmaceutical dosimetry should include robust biodistribution reporting J. Nucl. Med. 59 1507-9

Lassmann M et al 2008 EANM Dosimetry Committee series on standard operational procedures for pre-therapeutic dosimetry: I. blood and bone marrow dosimetry in differentiated thyroid cancer therapy Eur. J. Nucl. Med. Mol. Imaging 35 1405-12

Lassmann M and Eberlein U 2018 The relevance of dosimetry in precision medicine J. Nucl. Med. 59 1494-9

Lecoq P 2016 Development of new scintillators for medical applications Nucl. Instrum. Methods Phys. Res. A 809 130-9

Lin K J, Huang J Y and Chen Y S 2011 Fully automatic region of interest selection in glomerular filtration rate estimation from $99 \mathrm{mTc}$ DTPA renogram J. Digital Imaging 24 1010-23

Luster M et al 2008 Guidelines for radioiodine therapy of differentiated thyroid cancer Eur. J. Nucl. Med. Mol. Imaging $351941-59$

Marcu L, Bezak E and Allen B J 2018 Global comparison of targeted alpha vs targeted beta therapy for cancer: in vitro, in vivo and clinical trials Critical Rev. Oncol./Hematol. 123 7-20

Mathai A and Katiyar R 1996 A new algorithm for nonlinear least squares J. Math. Sci. 81 2454-63

Mather R L 1957 Gamma-ray collimator penetration and scattering effects J. Appl. Phys. 28 1200-7

Hubbell J and Seltzer S 2004 X-Ray mass attenuation coefficients NIST Standard Reference Database 12620899

O'Connor M K and Vermeersch C 1991 Critical examination of the uniformity requirements for single-photon emission computed tomography Med. Phys. 18 190-7

O'Connor M K, Cullen M J and Malone J F 1979 The value of a tracer dose in predicting the kinetics of therapeutic doses of $131 \mathrm{I}$ in thyrotoxicosis Br. J. Radiol. 52 719-26

Robinson A P et al 2016 The influence of triple energy window scatter correction on activity quantification for 177Lu molecular radiotherapy Phys. Med. Biol. 61 5107-27

Salvatori M and Luster M 2010 Radioiodine therapy dosimetry in benign thyroid disease and differentiated thyroid carcinoma Eur. J. Nucl. Med. Mol. Imaging $37821-8$

Segars W P et al 2010 4D XCAT phantom for multimodality imaging research Med. Phys. 37 4902-15

Siantar C L H et al 2002 Treatment planning for molecular targeted radionuclide therapy Cancer Biotherapy Radiopharmaceut. 17 267-80

Siegel J A et al 1999 MIRD Pamphlet No. 16: techniques for quantitative radiopharmaceutical biodistribution data acquisition and analysis for use in human radiation dose estimates $4037 \mathrm{~S}-61 \mathrm{~S}$

Sjögreen Gleisner K et al 2017 Variations in the practice of molecular radiotherapy and implementation of dosimetry: results from a European survey EJNMMI Phys. 4 1-20

Smith R N and Wilson G M 1967 Clinical trial of different doses of 131-I in treatment of thyrotoxicosis Br. Med. J. 1 129-32

Stabin M G and Brill A B 2008 State of the art in nuclear medicine dose assessment Semin. Nucl. Med. 38 308-20

Stokkel M P M et al 2010 EANM procedure guidelines for therapy of benign thyroid disease Eur. J. Nucl. Med. Mol. Imaging $372218-28$

Strigari L et al 2014 The evidence base for the use of internal dosimetry in the clinical practice of molecular radiotherapy Eur. J. Nucl. Med. Mol. Imaging 41 1976-88

Ting G, Chang C H and Wang H E 2009 Cancer nanotargeted radiopharmaceuticals for tumor imaging and therapy Anticancer Res. 29 4107-18

van Gils C A J et al 2016 Impact of reconstruction parameters on quantitative I-131 SPECT Phys. Med. Biol. 61 5166-82

Vija Racaru L et al 2017 Clinical outcomes 1 year after empiric 131I therapy for hyperthyroid disorders: real life experience and predictive factors of functional response Nucl. Med. Commun. 38 756-63

Yeong C H, Cheng M H and Ng K H 2014 Therapeutic radionuclides in nuclear medicine: current and future prospects J. Z. Uni. Sci. B 15 $845-63$ 